\title{
An Experimental Approach to Understanding the Optical Effects of Space Weathering
}

\author{
Sarah K. Noble*, Carlé M. Pieters \\ Brown University, Providence RI, 02912 \\ Ph: (401) 863-2417, Fax: (401) 863-3978 \\ Carle_Pieters@brown.edu \\ Lindsay P. Keller \\ NASA Johnson Space Center, Houston TX, 77058 \\ Ph: (281) 483-6090, Fax: (281) 483-1573 \\ Lindsay.P.Keller@nasa.gov
}

*Now at NASA Johnson Space Center, Houston TX, 77058

Ph: (281) 483-5077, Fax: (281) 483-1573

Sarah.K.Noble@nasa.gov

Manuscript Pages: 27

Figures: $\quad 14$

Tables: $\quad 2$

Submitted to Icarus January, 2007 
Proposed running head: Experimental Approach to Space Weathering

Editorial correspondence may be directed towards Sarah Noble:

NASA Johnson Space Center

Mail Code KR

Houston TX 77058

Sarah.K.Noble@nasa.gov

(281) 483-5077 


\section{Abstract:}

The creation and accumulation of nanophase iron $\left(\mathrm{npFe}^{0}\right)$ is the primary mechanism by which spectra of materials exposed to the space environment incur systematic changes referred to as "space weathering." The optical effects of this npFe ${ }^{0}$ on lunar soils are well documented. Space weathering though, should occur on the surface of any planetary body that is not protected by an atmosphere. There is no reason to assume that cumulative space weathering products throughout the solar system will be the same as those found in lunar soils. In fact, these products are likely to be very dependent on the specific environmental conditions under which they were produced. We have prepared a suite of analog soils to explore the optical effects of $n p F e^{0}$. By varying the size and concentration of $\mathrm{npFe}^{0}$ in the analogs we found significant systematic changes in the Vis/NIR spectral properties of the materials. Smaller $\mathrm{npFe} \mathrm{e}^{0}(<10 \mathrm{~nm}$ in diameter $)$ dramatically reddens spectra in the visible wavelengths while leaving the infrared region largely unaffected. Larger $\mathrm{nFe}^{0}$ ( $>40 \mathrm{~nm}$ in diameter) lowers the albedo across the Vis/NIR range with little change in the overall shape of the continuum. Intermediate $\mathrm{nFe}^{0}$ sizes impact the spectra in a distinct pattern that changes with concentration. The products of these controlled experiments have implications for space-weathered material throughout the inner solar system. Our results indicate that the lunar soil continuum is best modeled by $\mathrm{nFF}^{0}$ particles with bulk properties in the $\sim 15-25 \mathrm{~nm}$ size range. Larger $\mathrm{npFe}^{0}$ grains result in spectra that are similar in shape to the Mercury continuum. The continuum of S-type asteroid spectra appear to be best represented by small amounts of $n p F e^{0}$ that is similar to, but slightly smaller on average, than the $\mathrm{npFe}^{0}$ in lunar soils $(\sim 10-15 \mathrm{~nm})$.

Keywords: SURFACES, ASTEROIDS

MOON, SURFACE

MERCURY

SPECTROSCOPY 


\section{Introduction:}

"Space weathering" is a term used for a number of processes that act on any airless body exposed to the harsh space environment. The optical changes associated with space weathering are largely initiated by two of these processes, micrometeorite bombardment and charged particle irradiation. Both processes create nanophase iron $\left(\mathrm{npFe}^{0}\right)$, nanometer-scale particles of metallic iron distributed in amorphous silicate. In lunar soils, $\mathrm{npFe}^{0}$ is found in two places: throughout agglutinitic glass and on soil grains in vapor/sputter deposited and irradiated rims. The average size of these iron particles in $\mathrm{npFe}^{0}$-bearing rims is observed to be about $3 \mathrm{~nm}$ in diameter, though they range from roughly 1 to $15 \mathrm{~nm}$ (Keller and Clemett, 2001). The $\mathrm{npFe}^{0}$ particles in agglutinates are considerably larger (Keller and Clemett, 2001) and come in a wider range of sizes up to several hundred nanometers (Housley et al., 1973; James et al., 2001).

The optical consequences of $\mathrm{nFFe}^{0}$ on lunar soils have been long recognized and are well documented (e.g. Pieters et al., 1993, 2000; Hapke, 2001; Noble et al., 2001). It is known that these optical effects, the so-called "space weathering continuum," change with the amount of $\mathrm{npFe}^{0}$ present (Noble et al., 2001); however, minimal work has been done to explore the effects of $n \mathrm{pFe}^{0}$ size on the optical properties. Based on a combined microspectroscopic/TEM study, Keller et al. (1998) suggested that smaller $\mathrm{npFe}^{0}$ particles $(\leq 5 \mathrm{~nm})$ would result in the spectral reddening that is associated with space weathering, but larger particles would cause only darkening and not reddening. Also, Britt and Pieters (1994) found that inclusion of larger (micron-scale) particles of iron result in wavelength independent darkening throughout the visible/near-infrared (Vis/NIR) wavelengths.

The Moon is currently the only body from which we have direct and documented samples of space weathered material and thus, nearly all of our knowledge of space weathering comes from 
lunar samples. Space weathering products though, should be created on any body exposed to the space environment. There is no reason to believe that space weathering products will be the same everywhere (Hapke, 1986, 2001); as the space environment varies, the products of space weathering should change as well. Composition, distance from the sun, temperature, gravity, existence of a magnetic field, rate and velocity of impacts, and many other factors will all play a role in how space weathering manifests itself on any given body. Thus, while $\mathrm{npFe}^{0}$ may be created on bodies other than the Moon, there is no guarantee that the size or distribution of $\mathrm{nFFe}^{0}$ particles will be the same. It is therefore important that we understand how the optical properties of a material are affected by the size of $\mathrm{nFFe}^{0}$ particles. We have created a space weathering analog material for which $\mathrm{nFe}^{0}$ size can be controlled and allow us to quantify the effect of size and number density of $\mathrm{nFe}^{0}$ on optical properties. This systematic approach allows us to deconvolve and isolate the effects of $\mathrm{nFe}^{0}$ from other soil properties.

\section{Methods:}

Analog soils were prepared using commercial chromatography grade silica gel powders. The amorphous silica gel powders are extremely porous and have a narrow distribution of pore sizes. The four powders used were manufactured by two different companies; the gels with 2.3, 6 and $25 \mathrm{~nm}$ pores (the SG2, SG6 and SG25 suites, respectively) are manufactured by GRACEDavidson, while the $50 \mathrm{~nm}$ pore gels (the SG50 suite) are produced by Mallinckrodt Baker Inc (Table 1). Also, the gel powders do not all come in the same particle size ranges. Table 1 lists the range of particle sizes for each specified pore size used as starting material.

The gel powders were impregnated with ferric nitrate solutions of various normalities $(0.001$ $1.0 \mathrm{~N}$ ) to produce a range of iron concentrations (Morris et al., 1989; Allen et al., 1996). It is 
difficult to control exactly how much iron is introduced into each sample, i.e. samples exposed to the same normality solutions may acquire different total iron concentrations. Rather than attempt to create samples of each pore size with identical iron concentrations, we focused on creating a suite of samples for each of the four powders that provided the widest range of optical properties possible, regardless of the exact amount of iron required to achieve the results. The actual amount of iron in each sample was quantitatively determined by later measurements (see below).

The ferric-nitrate impregnated gels were dried in air before calcining in air at $\sim 500^{\circ} \mathrm{C}$ for 40 80 hours, producing faintly-orange to deep reddish-brown samples with varying amounts of nanophase hematite $\left(\mathrm{Fe}_{2} \mathrm{O}_{3}\right)$ in the pores. Subsequently, the samples were placed in a furnace at $850-900^{\circ} \mathrm{C}$ under reducing conditions (pure flowing hydrogen) for approximately 4 hours and then cooled while hydrogen flow was maintained.

After reduction, some of the samples took on a "salt and pepper" appearance as individual grains within each sample acquired varying amounts of iron. This effect was most prominent in the suite with the smallest pore sizes (SG2). The SG6 suite also showed some variation within each sample, as illustrated by the backscatter image in Fig. 1. Grains of the large pore size gels (SG25 and SG50) were more uniform in appearance to both the naked eye and in backscatter images.

\section{Analytical Methods and Results:}

\section{Neutron Activation Analysis:}

Iron contents of the prepared samples were measured via neutron activation analysis (by D. Mittlefehldt). Roughly 300 to $1300 \mathrm{mg}$ of each sample was weighed and placed into a glass vial. The amount of sample used was roughly the same volume to minimize geometry effects. The 
samples were dried overnight at $250^{\circ} \mathrm{C}$ to devolatilize the silica gel, cooled to room temperature in a desiccator, and then reweighed. The dried samples were transferred to polyethylene vials and heat-sealed. Four samples of roughly $20 \mathrm{mg}$ each of the international standard rocks IF-G (iron formation) and Mica-Fe (biotite) were weighed for use as standards and controls. Each was mixed with crushed ultra-pure silica glass to closely reproduce the geometry of the larger unknown samples. The samples were loaded into four irradiation cans, with one standard and one control in each can, at the Texas A\&M University Nuclear Science Center. The cans were irradiated for 4 hours at a thermal neutron flux of $6.6 \times 10^{12} \mathrm{n} \mathrm{cm}^{-2} \mathrm{sec}^{-1}$, and then allowed to decay for six days. The samples were returned to Johnson Space Center, the vial exteriors were cleaned, and counted in the Gamma-ray Spectroscopy facility. The samples were counted on two intrinsic Ge high efficiency detectors in the low-level counting room. Data reduction was done following normal JSC procedures (Mittlefehldt, 1994). The results are given in Table 2.

\section{Transmission Electron Microscopy:}

TEM images were obtained on multiple samples from each suite. The TEM analysis was performed on a Phillips 420 at Brown University and a JEOL 2000FX at Johnson Space Center. Samples were prepared by embedding grains of the silica gel in epoxy and thin sectioning with an ultramicrotome to obtain $\sim 70 \mathrm{~nm}$ thick sections. It was generally found that the $\mathrm{npFe}^{0}$ particles were well dispersed throughout the grains, although occasionally concentrations were

observed near grain edges. The $\mathrm{nFe}^{0}$ particles are typically round, but some more angular shapes are observed. Not uncommon, particularly among the larger particles, are squares with facets at the corners; this is the equilibrium shape (Wulff form) of $\alpha$-Fe (e.g. Zangwill, 1988). 
Electron diffraction data were obtained for representative samples from all four suites. The silica substrate resulted in diffuse rings, typical of an amorphous material. There were no observed diffraction spacings due to any of the crystalline silica polymorphs, which indicates that the substrate remained amorphous even after heating. The $\mathrm{nFe}^{0}$ was found to be dominantly $\alpha-\mathrm{Fe}$, though $\gamma$-Fe was identified as well. There does not appear to be any clear trend with size of iron and preference for one polymorph over the other. We observed no electron diffraction evidence for oxidized forms of $\mathrm{Fe}$ (e.g. FeO, Fe3O4, Fe2O3).

For each pore size a range of $\mathrm{nFe}^{0}$ sizes were observed (Table 1). The average $\mathrm{npFe}^{0}$ size within each size suite was significantly larger than the manufacturer's stated pore size of the silica gels, with the exception of the $50 \mathrm{~nm}$-pore suite (SG50), which averaged somewhat smaller than its stated pore size. The SG50 suite also contained the widest range of npFe ${ }^{0}$ sizes, up to $200 \mathrm{~nm}$, but the average size was around $40 \mathrm{~nm}$, not much larger than the SG25 suite. A similar size range was observed for samples with $100 \mathrm{~nm}$ pores. It appears that it is difficult to create larger $\mathrm{nFe}^{0}$ with this method and that, at this point, $\mathrm{npFe}^{0}$ size is no longer being controlled by pore size. All attempts to create larger $\mathrm{npFe}^{0}$ by this method proved unsuccessful. As the TEM sections are only $\sim 70 \mathrm{~nm}$ thick, the size distributions observed will be skewed slightly towards smaller sizes as some of the $\mathrm{nFe}^{0}$ spheres will be cut at various cross-sections, particularly for the larger iron suites, thus we expect the true average sizes for the SG25 and SG50 suites are likely slightly larger than observed.

Representative TEM bright field images from microtomed thin sections of each of the four powders are shown in Fig. 2. While the different original pore sizes clearly affected the final size of the $\mathrm{nFe}^{0}$, the particles were not strictly confined to the pores. The reason for this is unknown, though one possibility is that since the pores are so well connected the original 
hematite may have been able to occupy several connected pores, which, after reduction, would result in a larger $\mathrm{npFe}^{0}$ particle.

In addition to the nominal range of $n \mathrm{pFe}^{0}$ sizes produced for each suite (Table 1), occasional large particles (up to several hundred nanometers) were observed in all samples regardless of pore size. These large particles often occurred near edges where pores did not confine them, though some are also found in grain interiors. Several examples of these large particles are shown in Fig. 3.

\section{Mossbauer Spectra:}

Representative samples from the SG2, SG6 and SG25 suite were analyzed by Mössbauer spectroscopy to confirm the iron structure identified in TEM. Mossbauer spectra were acquired at $295 \mathrm{~K}$ using a source of $50-30 \mathrm{mCi}^{57} \mathrm{Co}$ in $\mathrm{Rh}$ on a WEB Research Co. model W100 spectrometer equipped with a Janus closed-cycle He refrigerator. Run times were 24 hours. The sample from the SG25 suite was found to have a well-ordered iron spectrum. As shown in Fig. 4, it is nearly indistinguishable from an $\alpha$-Fe foil. The SG2 and SG6 samples also display longrange ordered $\alpha-\mathrm{Fe}$, but there is an additional doublet in both spectra that may be due to the smaller grain size $\mathrm{Fe}$ or to the presence of $\gamma$-Fe. There is no evidence for the presence of hematite or other iron oxides. Therefore, based on detection limits for the instrument, if present, these oxides would have to constitute less than $1 \%$ of the total iron in the samples.

\section{Visible/Near-Infrared Spectra:}

Bidirectional Vis/NIR spectra for all samples were measured in RELAB at Brown University at $\mathrm{i}=30^{\circ}, \mathrm{e}=0^{\circ}$. The reflectance spectra for the four experimental suites of samples with $n p F e^{0}$ 
are shown in Fig. 5. For each of the four suites of silica gels, a null sample was included in the suite in which no iron was added, though the samples were exposed to the same heating and reducing conditions as the others (see Appendix). These null samples, the highest albedo spectrum in each suite, contain only the trace amounts of iron that are inherent to the silica gel (Table 2). The remaining samples contain increasing amounts of iron and exhibit a correlated decrease in reflectance.

Silica gels are commonly used as a desiccant, and given the high surface area of these materials, it is not surprising that adsorbed water was an issue with all of the samples. In general, the smaller pore sizes adsorbed more water than the larger pores. Absorbed water is shown by the $\mathrm{OH}$ absorption peaks, most prominent at $\sim 1350 \mathrm{~nm}$ and smaller at $\sim 950$ and 1250 $\mathrm{nm}$. The strength of these bands decrease with increasing iron content as their signature is masked by the absorption properties of the iron particles. The features are rather sharp absorption bands and, in general, they do not affect the continuum shape over the wavelength region of interest. Hydroxyl and water bands do become more prominent at longer wavelengths $(>2 \mu \mathrm{m})$ and begin to interfere with the continuum, so all of the spectra were truncated at 1800 $\mathrm{nm}$ to avoid that interference and facilitate comparisons among samples.

Allen et al. (1996) were the first to use silica gels as an optical analog for lunar space weathering products. Their study concentrated on gels similar to those used here with $6 \mathrm{~nm}$ pores. Our spectra of the SG6 suite (Fig. 5b) are in general agreement with their results. The spectral trends seen in the SG6 suite also correspond with the theoretical modeling results of Hapke (2001) who modeled the Vis/NIR optical properties of $10 \mathrm{~nm}$ diameter $\mathrm{npFe}^{0}$-bearing coatings on $10 \mu \mathrm{m}$ grains. 
Using the SG6 suite as an example (Fig. 5b), the spectral trends with increasing $n p F e^{0}$ content exhibit four distinct stages: (1) Initially, with small amounts of $\mathrm{npFe}^{0}(\sim 0.02 \mathrm{wt} \%)$ a steep curvature develops in the visible region while longer wavelengths $(>750 \mathrm{~nm})$ remain relatively unaffected. (2) With additional iron $(>0.1 \mathrm{wt} \%)$, the spectra become very red (reflectance increases with increasing wavelength), while the curvature becomes less extreme and extends to longer wavelengths. (3) As more iron is added (>0.2 wt \%), the spectra lose their curvature and become increasingly linear. (4) Finally, at the highest iron concentrations ( $>1 \mathrm{wt}$ $\%$ ), the spectra become increasingly dark with a slightly convex shape.

The SG2 suite spectra, shown in Fig. 5a, display significant differences from the SG6 suite. Again, the brightest spectrum contains only trace amounts of iron, while the remaining samples increase in iron concentration as reflectance decreases. The $\mathrm{OH}$ bands are more prominent for this suite, and more problematic. Adsorbed water is affecting the continuum of some of the spectra at wavelengths beyond the $1350 \mathrm{~nm} \mathrm{OH}$ band causing an apparent reduction in the albedo. The introduction of this very small size $(5-15 \mathrm{~nm}) \mathrm{npFe}^{0}$ results in a dramatic curvature in the visible region, much stronger than what was seen in the SG6 suite with larger $\mathrm{npFe}^{0}$. Adding additional $\mathrm{npFe}^{0}$ results in an overall decrease in the albedo, but does not produce the strong reddening in the infrared that was seen in the SG6 suite. By scaling the SG2 suite to unity at $1300 \mathrm{~nm}$ (Fig. $5 \mathrm{a}$ ), it becomes clear that wavelengths greater than $\sim 700 \mathrm{~nm}$ remain virtually unaffected by the $n \mathrm{pe}^{0}$ until very high concentrations are reached. Only the two highest iron samples (those with $>1 \mathrm{wt} \% \mathrm{npFe}^{0}$ ) show any redness in the infrared.

Larger $\mathrm{nFFe}^{0}$ particles also have unique optical properties. The reflectance spectra for the SG25 suite are shown in Fig. 5c. With small amounts of these larger iron particles $(<0.1 \mathrm{wt} \%)$, the spectrum is only slightly curved in the visible region and exhibits a significant red slope into 
the near infrared. This spectrum is higher in albedo, but similar in shape, to the "stage 2" of the SG6 suite. Intermediate iron contents $(\sim 0.1-1.0 \mathrm{wt} \%)$ result in very linear, very red-sloped spectra. Additional $\mathrm{npFe}^{0}\left(>1 \mathrm{wt} \% \mathrm{npFe}^{0}\right)$ in this size range results in spectra with an increasingly dark and more convex shape.

The reflectance spectra of the SG50 suite, the largest $\mathrm{npFe}^{0}$ particles in our study, are shown in Fig. 5d. Although albedo is strongly affected, the overall shapes of these spectra show little change as a function of increasing $\mathrm{npFe}^{0}$ content. The lowest iron-content sample $(0.02 \mathrm{wt} \%$ $n \mathrm{pFe}^{0}$ ) is nearly linear with a slight red slope. Adding additional $\mathrm{npFe}^{0}$ particles results in a progressively lower albedo and a slight increase in the red slope at wavelengths greater than $\sim 1000 \mathrm{~nm}$. The most iron-rich sample $\left(1.89 \mathrm{wt} \% \mathrm{npFe}^{0}\right)$ displays the convex continuum that was observed in the other suites.

\section{Discussion:}

\section{Effects of npFe $^{0}$ size:}

The size of the $\mathrm{npFe}^{0}$-particles dramatically influences the spectra of these samples. To illuminate these differences, spectra of samples with similar iron contents from each of the four pore-size suites are shown in Fig. 6. Even low concentrations $(0.02 \mathrm{wt} \%)$ of $\mathrm{npFe}^{0}$ cause dramatic spectral effects (Fig. 6a). The SG2, SG6 and SG25 suite all show a substantial reddening in the visible region. In addition, the SG6 and SG25 spectra also display reddening throughout the near-IR. In addition, the SG6, SG25 and SG50 samples show a dramatic reduction in albedo. Recall that the individual size of particles in the SG50 gels is smaller than the others (Table 1). As a result, the SG50 sample appears relatively bright in relation to the other suites, although it is still considerably darker than the no-iron-added sample from that suite 
(Fig. 5d). The second plot (Fig. 6b) contains reflectance spectra of samples with $\sim 0.2$ wt $\%$ $n \mathrm{nFe}^{0}, 10 \mathrm{x}$ as much iron as in the previous plot (Fig. 6a). Here the differences between the four sizes of $\mathrm{npFe} \mathrm{e}^{0}$ are most prominent. All four spectra have considerably lower albedos compared to the results for $0.02 \mathrm{wt} \% \mathrm{npFe}^{0}$ (Fig. 6a). The continuum shape for the SG2 sample has not changed significantly from the $0.02 \mathrm{wt} \%$ sample, in that there is still a sharp curvature in the visible region and almost no change in the near-IR. The SG6 sample has become redder throughout the Vis/NIR relative to the other samples, though it still displays a curvature towards the visible region. The SG25 sample, by contrast, has lost its curvature and is now linear and very red. The SG50 sample is still flat in the visible region, but shows a slight reddening at longer $(>1000 \mathrm{~nm})$ wavelengths.

A clear correlation can be observed between the $\mathrm{npFe}^{0}$ size and the wavelengths that are affected. The smallest $n p \mathrm{Fe}^{0}$ particles strongly affect the visible region of the spectrum. At longer wavelengths, though, the iron in the SG2 suite appears to have little, if any, effect. For larger particles, the visible region is less affected, while influence on the near-IR grows stronger. The SG6 and SG25 suites show a dramatic reddening in the near-IR region. At $\mathrm{npFe}^{0}$ sizes greater than $\sim 40 \mathrm{~nm}$ (i.e. the $\mathrm{SG} 50$ suite), there is a distinct change as all wavelengths are similarly affected and the greatest change as a function of $\mathrm{npFe}^{0}$ concentration is a reduction in albedo.

Based on the results of the SG50 suite, the occasional anomalously large particles (e.g. Fig. 3), which occur in all samples, would likely cause a general darkening of the spectra across all wavelengths of interest, and thus should have no significant effect on the shape of the continua. Further, since they are observed in all sample suites, any minor darkening effects attributed to the anomalously large particles would be shared by all samples and thus should not affect 
comparisons between suites. Nevertheless, their presence complicates a quantitative comparison of albedo.

\section{Mixing issues:}

In addition to size and abundance, the microscale distribution of $\mathrm{npFe}^{0}$ is another important variable that depends on environment. In the lunar case, the shape of the space weathering continuum appears dependent only on the total amount of $\mathrm{nFe}^{0}$ present, independent of how much $\mathrm{FeO}$ was in the soil initially (Noble et al., 2001). The standard measure of lunar soil maturity is $\mathrm{I}_{\mathrm{s}} / \mathrm{FeO}$ (Morris, 1978), i.e. the amount of $\mathrm{npFe}^{0}$ (measured by $\mathrm{I}_{\mathrm{s}}$ ) normalized to the total amount of $\mathrm{Fe}$ in the sample (measured by $\mathrm{FeO}$ ). Consequently, it is possible for a mare and highland soil with different quantities of $\mathrm{FeO}$ to have different maturities but similar quantities of $\mathrm{npFe}^{0}$. This pair will have similar continuum shapes although the mare soil, being less mature, will likely have fewer rims and agglutinates with somewhat more densely packed $n p F e^{0}$ than the more mature highland soil.

In more extreme cases, however, $\mathrm{npFe}^{0}$-distribution might become an important factor. This can be demonstrated by synthetic mixtures of our SG6 analog soils with the highest iron content (2.1 wt \% iron) and the SG6 no-iron-added sample (Fig. 7). The resulting spectra of these mixtures vary largely in albedo while the general shape of the continua remains virtually unchanged from the high-iron end-member spectrum. The continua of these spectra for mixed samples are behaving as would be expected for large $\mathrm{npFe}^{0}$ particles. However, TEM analysis confirms that this is not the case; the size of $\mathrm{nFe}^{0}$ in this sample is on average the same as was observed in lower concentration samples. It is our hypothesis that the $\mathrm{nFe}^{0}$ particles are so densely packed here that, optically, they are behaving as larger particles. 
Lunar soils have been observed to contain a wide range of $\mathrm{npFe}^{0}$ sizes. In particular, the $n p \mathrm{Fe}^{0}$ in vapor/sputter deposited and irradiated rims is generally much smaller than the $\mathrm{npFe}^{0}$ found in the interior of agglutinates. Mixing of smaller and larger particles results in a roughly linear mixing of the spectra for Vis/NIR wavelengths. In Fig. 8, spectra are shown that contain roughly equal amounts of iron $(\sim 0.2 \mathrm{wt} \%)$ from the SG6 and SG25 suites. Because the spectrum of this mixed sample was measured several months after the originals, there is more adsorbed water and that appears to be affecting the continuum, particularly at wavelengths greater than $\sim 1300 \mathrm{~nm}$, reducing the reflectance. This unfortunately complicates the interpretation, as it is difficult to separate the water effects. When the two are mixed together in

roughly equal proportions, the resulting spectrum falls roughly equidistance between the endmembers in the visible region, then slightly closer to the SG25 sample up to $\sim 1300 \mathrm{~nm}$ where the continuum is highly influenced by the adsorbed water and a good comparison is no longer possible. We conclude that the continuum shape of a given spectrum is generally a reflection of the average $\mathrm{npFe}^{0}$-size for that sample, though larger iron may have slightly more influence.

\section{Implications:}

A major objective of this work was to quantify the effects of $\mathrm{npFe}^{0}$ size, density, and distribution and to use that data to better understand space weathering. It is useful to compare our experimental samples to naturally space-weathered materials and surfaces. The possible implications of our results for understanding the products of space weathering created in different environments throughout the inner solar system are explored below. 


\section{The Moon:}

In Fig. 9 we compare the reflectance spectra of several lunar soils to samples of the SG2, SG6 and SG25 suites. (The $\sim 1 \mu \mathrm{m}$-band in the lunar samples is due to pyroxene and olivine in those samples.) Compared to the SG2 suite, the soils are far too red in the near-IR and not red enough in the visible region. The natural soils show the greatest similarity to the SG6 suite, though when examined in detail, the soils are slightly redder in the near-IR, and slightly less red in the visible region. This trend suggests that the $\mathrm{npFe}^{0}$ in the lunar soils is on average slightly larger than the SG6 suite. However, the lunar $\mathrm{npFe}^{0}$ must be smaller than the $\mathrm{npFe}^{0}$ in the $\mathrm{SG} 25$ suite as the soils are not red enough in the near-IR and are too red in the visible region compared to those analogues. The lunar space weathering continuum, then, appears to be created by $n p \mathrm{Fe}^{0}$ that is slightly larger than the SG6 suite $\mathrm{npFe} \mathrm{e}^{0}$, on the order of $\sim 15-25 \mathrm{~nm}$ on average.

The average size of npFe ${ }^{0}$ observed in lunar soils rims with TEM is only about $3 \mathrm{~nm}$ (Keller and Clemett, 2001). However, agglutinates and agglutinate fragments comprise a significant fraction of lunar soils and contain $\mathrm{nFe}^{0}$ that is considerably larger than the iron found in rims (Keller and Clemett, 2001; James et al., 2001, Fig. 10). It was once thought that agglutinates were the main cause of the spectral alterations that are referred to as "space weathering," from the original (erroneous) idea of vitrification-darkening (e.g. Conel and Nash, 1970; Adams and McCord, 1973) to the discovery of the role of $\mathrm{npFe}^{0}$ (Hapke et al, 1975; Cassidy and Hapke, 1975). In recent years, however, the role of agglutinates in the weathering process has been often overshadowed by the focus on $\mathrm{nFFe}^{0}$-bearing rims. A major finding of this work is the observation that it is in fact the combination of both the small $n p F e^{0}$ in the rims and the larger $n p F e^{0}$ in agglutinates that results in the unique continuum of lunar soils. 


\section{Mercury:}

Mercury's reflectance spectrum has a significant red slope and is essentially free of detectable absorption bands in the Vis/NIR. The lack of absorption bands, particularly the lack of a $1 \mu \mathrm{m}$ pyroxene absorption band, has been cited as evidence that Mercury's surface is devoid of iron (e.g. Vilas, 1985). However, the red slope has been attributed by others (McCord and Clark, 1979; Hapke, 2001) to space weathering products. Hapke (2001) suggests that the red slope represents soils with 2-3 wt $\% \mathrm{FeO}$. Independent of the Vis/NIR data, microwave data also suggests that Mercury's surface is very iron-poor, containing only about 1 wt $\% \mathrm{FeO}$ (Jeanloz, 1995).

Mercury's surface must contain some iron: even if the native surface rocks are completely devoid of iron (metallic and ferrous), the iron brought in by meteorite and comet impacts should accumulate in the soil and would be enough to affect the optical properties. From trace element abundances, iridium in particular, it has been estimated that $1-4 \%$ of the lunar soil is meteoritic contamination (Haskin and Warren, 1991). The flux of impactors on Mercury is nearly six times the lunar flux (Cintala, 1992). Depending on the composition of the impactor population (for example, the ratio of comets to asteroids is likely greater on Mercury - Hartmann, 1977), the surface soils may contain as much as $5-20 \%$ meteoritic components. Iron brought in by meteorites therefore, could account for as much as $1-5 \mathrm{wt} \% \mathrm{FeO}$ in the regolith. The addition of meteoritic components necessarily requires that the iron content of the Mercurian regolith must be measurably greater than zero.

We have shown in earlier sections that it takes little $\mathrm{npFe}^{0}(>0.02 \mathrm{wt} \%)$ to dramatically affect the spectral properties of a material. For lunar soils, we have found the effects of very small amounts of $\mathrm{nFe}^{0}$ are significantly different than for larger amounts (Noble et al., 2004). 
Like the SG6 suite, small amounts of $\mathrm{npFe}^{0}$ in lunar soils result in a steeply curved spectrum with the visible region much more strongly affected than longer wavelengths. Only at relatively high iron concentrations does the continuum become linear. After removal of the thermal component, telescopic spectra of Mercury's Vis/NIR spectrum is remarkably linear (e.g. Sprague et al., 2004). If Mercury's surface contains so little iron, and by extension, very little $n p F e^{0}$, then its linear spectrum does not fit the lunar model. Mercury's spectrum with its red and linear slope is more compatible with our results for the SG25 suite where a linear continuum results from small amounts of larger npFe ${ }^{0}$ particles.

Blewett et al. (1997) measured the continuum slope of several Mercury spectra from 745 to $1078 \mathrm{~nm}$ (scaled to 1 at $1.02 \mu \mathrm{m}$ ) and found the slope to fall in the range of $0.65-0.7 \mu \mathrm{m}^{-1}$, generally redder than several telescopic measurements of lunar anorthosites, which ranged from 0.3-0.59. An analog sample from the SG25 suite containing $0.2 \mathrm{wt} \%$ iron, was found to have a continuum slope of 0.88 over the same region when similarly scaled. Two Earth-based telescopic spectra of Mercury (Vilas, 1984) are compared to several analog spectra in Fig. 11. Similar to the lunar soils, the spectrum of Mercury does not compare well with the SG2 suite because it is far too red in the near-IR and not red enough in the visible region. Though the SG25 sample is arguably the better fit, the Mercury spectra are similar in shape and redness to samples from both the SG6 and SG25 suites. Closer inspection however, reveals important discrepancies. The Mercury spectra do not match well the curvature of the lower-iron SG6 gel sample $\left(0.19 \mathrm{wt} \% \mathrm{npFe}^{0}\right)$ in the visible region. The higher-iron SG6 sample $\left(0.30 \mathrm{wt} \% \mathrm{npFe}{ }^{0}\right)$ is by comparison more linear, and thus more similar to the Mercury spectra, however, it requires a very large amount of $\mathrm{nFe}^{0}$. Recall that the lunar soil spectra were closest in shape to silica gels with roughly $0.1-0.2 \mathrm{wt} \% \mathrm{npFe}{ }^{0}$ (Fig. 10). This implies that if $\mathrm{npFe}^{0}$ on Mercury and the 
Moon is of similar size, then Mercury's soils would have to be more $n p \mathrm{pe}^{0}$-rich than even the lunar mare. If however, the $\mathrm{npFe}^{0}$ particles are larger on Mercury (more similar to the SG25 suite), then less is required for the optical effects observed. Though, even with larger iron, our results predict that Mercury's surface is still more $\mathrm{nFe}^{0}$-rich than typical lunar highlands.

\section{Why is the $n p F e^{0}$ on Mercury larger than on the Moon?:}

The space weathering environment at Mercury differs substantially from the Moon. One major difference is the diurnal variation of surface temperature $\left(\sim 300^{\circ} \mathrm{C}\right.$ for the Moon, $\sim 700^{\circ} \mathrm{C}$ on Mercury). The high temperatures on the Mercurian surface may result in larger $\mathrm{npFe}^{0}$, particularly near the equator, due to Ostwald ripening processes (Noble and Pieters, 2003). Therefore, one might predict that areas near the equator would have a lower albedo, and in fact, recent Earth-based telescopic spectra suggest that the Mercurian continuum may in fact be darkest near the equator and become redder with increasing latitudes (Warell, 2002), though no latitudinal variations have been seen in Mariner 10 data (Hapke, 1977).

In addition to the extreme temperature regime, Mercury also has an increased impactor flux of micrometeorites and its location in the solar system and greater mass result in higher velocity impacts. These factors combine to make Mercury much more efficient than the Moon at creating both melt and vapor. Per unit area, impacts on Mercury are expected to produce $13.5 \mathrm{x}$ the melt and 19.5x the vapor than is produced on the Moon (Cintala, 1992). Agglutinitic glass-like deposits and vapor-deposited coatings should be created significantly faster and more efficiently on Mercury than on the Moon.

As noted earlier, agglutinitic glass contain significantly larger $\mathrm{npFe}^{0}$ than is found in $\mathrm{nFe}^{0}$ bearing rims (Keller and Clemett, 2001; James et al., 2001). It has been proposed that the reason 
for this disparity in $\mathrm{nFFe}^{0}$ size is that as grains with $\mathrm{npFe}^{0}$-bearing rims melt to form agglutinates, the reduced iron in the rims can coalesce to form larger particles (Taylor et al., 2001). The increased melting/vaporization rates on Mercury suggest that more recycling should be occurring there than is observed on the Moon. Grains will melt and vaporize to form agglutinates and rims, and these will be re-melted and re-vaporized to form new generations of agglutinates and rims. With each cycle comes the opportunity to create ever-larger npFe ${ }^{0}$ Pulse laser heating experiments to simulate micrometeorite bombardment show that repetitive heat pulses do indeed result in growth of $\mathrm{npFe}^{0}$ particles (Sasaki and Kurahashi, 2004). While vaporization/condensation will continually be creating new (i.e. small) $\mathrm{npFe}^{0}$, the average $n p F e^{0}$ size on Mercury will evolve to larger sizes than the Moon.

Our data indicate that, even assuming larger $n \mathrm{pFe}^{0}$ grains, there is considerable $n p \mathrm{Fe}^{0}$ in the Mercurian regolith ( $\sim 0.2 \mathrm{wt} \%)$, more than the typical amounts seen in lunar highlands soils (Fig. 9). This does not necessarily require that the Mercurian surface has as high a bulk FeO content as the lunar highlands $(\sim 5-7 \mathrm{wt} \%)$. Because of the significantly greater weathering rates, it is expected that, for a mature soil on Mercury, a much larger percentage of the total iron will exist in a reduced form (i.e. as $\mathrm{npFe} \mathrm{e}^{0}$ ) than is seen in lunar soils, where only a minor, though highly variable, fraction of the total iron is reduced through weathering (Keller et al., 1999).

\section{Asteroids:}

There is a wide variety of asteroid compositions and environments in our solar system, and these differences will almost certainly influence the products of space weathering (see Chapman, 2004 for a review of space weathering on asteroids). Here we will focus only on S-type asteroids, the class for which lunar-like weathering processes have long been suggested. It is 
worth pointing out, however, that even among S-type asteroids, there is thought to be considerable compositional variation, which may influence weathering on any particular asteroid. In addition, the near-Earth environment is likely to differ substantially from the mainbelt environment in terms of solar wind flux, and impact rates and velocities, which may also affect weathering products.

There has been much debate in the asteroid and meteorite communities about the existence and nature of space weathering. As the concept of space weathering has become more accepted (e.g. Chapman, 2004), it has been repeatedly suggested that the spectral differences between Stype asteroids and ordinary chondrites can be accounted for by very small amounts of lunar-like space weathering products (i.e. $\mathrm{npFe}^{0}$-bearing rims) (e.g. Binzel et al., 1996; Pieters et al., 2000; Hapke, 2001; Noble et al., 2004, 2006). The results of the current study also support this hypothesis. In Fig. 12 are shown reflectance spectra of two near-Earth S-type asteroids (Binzel et al., 2001) compared to samples from the SG2, SG6, and SG25 suites. These spectra, like those from the Moon and Mercury, do not compare well to the SG2 suite, they are too red in the near-IR and do not share the dramatic curvature of the SG2 suite in the visible region. The SG25 suite fits reasonably well in the near-IR, but the asteroid spectra are too red in the visible region. Like the lunar soils, the continua shapes of S-type asteroid spectra are best approximated by the SG6 suite. Here though, the asteroid spectra display continua which are slightly less red in the near-IR than the SG6 samples. This suggests that the size of $n p F e^{0}$ particles in asteroid regolith is slightly smaller on average than the iron in the SG6 suite, and therefore, smaller than the average lunar $n p \mathrm{Fe}^{0}$ as well. We predict that the average $n p \mathrm{Fe}^{0}$-size for these S-type asteroids must be roughly $10-15 \mathrm{~nm}$. 
Why might the npFe ${ }^{0}$ on the asteroids be smaller than on the Moon?:

One explanation for this size difference of $\mathrm{nFFe}^{0}$ may be a lack of agglutinates in asteroid soils. Several studies of regolith breccia meteorites have confirmed the rarity of agglutinates in asteroid regoliths (e.g. Basu and McKay, 1983). As discussed above, in lunar soils npFe ${ }^{0}-$ particles in weathering rims are significantly smaller than those found in agglutinates (Keller and Clemett, 2001). Ergo, fewer agglutinates relative to $\mathrm{npFe}^{0}$-bearing rims would result in a population of $\mathrm{nFFe}^{0}$ that are on average smaller in diameter than lunar soils.

Smaller average size of $\mathrm{npFe}^{0}$ particles in asteroid soil would account for the lower redness in the near-IR region. The particles still must be considerably larger than the $3 \mathrm{~nm}$ average of particles in lunar soil rims, however, since the asteroid continuums are much closer to the SG6 suite than the SG2 suite. This suggests that agglutinates may be more common in asteroid regoliths than is predicted by what is seen in regolith breccia meteorites, or the formation of $\mathrm{npFe}^{0}$ in asteroidal weathering rims results in the average size being larger than for the lunar case. Alternatively, Horz et al. (2005) suggests that nano-scale Fe-Ni and troilite particles created during impact shock and melting may be responsible for the optical changes observed on asteroids rather than the rims and agglutinates of lunar-style weathering. However, the results of the impact experiments carried out by Horz et al. suggest that this process may create particles which average considerably larger than our prediction of 10-15 nm.

\section{Conclusions:}

The creation of analog space weathering products has allowed us to explore the effects of varying both size and concentration of nanophase iron on visible and near-infrared reflectance 
spectra. These effects were found to be quite dramatic: Vis/NIR reflectance spectra vary in significant but predictable ways due to the size and amount of $\mathrm{npFe}^{0}$.

The size of $n p \mathrm{Fe}^{0}$ has a distinct impact on the wavelengths influenced. Smaller $n p F e^{0}(>10$ $\mathrm{nm}$ in diameter) more strongly affects the visible wavelengths, dramatically reddening those wavelengths while leaving the infrared largely unaffected. In contrast, $\operatorname{larger} n \mathrm{nFe}^{0}(>40 \mathrm{~nm}$ in diameter) impacts the entire Vis/NIR wavelength range, resulting in significantly lower albedos with little change in the continuum shape. Intermediate sizes, like those found in lunar soils, follow a unique pattern as $\mathrm{npFe}^{0}$ concentration is increased: beginning with an initial steep curvature in the visible region. Then the curvature becomes less extreme and extends to longer wavelengths as reddening increases. Finally the spectra lose their curvature and become increasingly dark and linear with a strong red slope.

By analyzing the specific spectral trends of several suites of analog materials with different $\mathrm{npFe}^{0}$ sizes, we have found evidence that suggests that $\mathrm{nFe}^{0}$ sizes vary throughout the inner solar system. Although the same fundamental space weathering processes act on all airless bodies, average $\mathrm{npFe}^{0}$ sizes on the asteroids appear to be slightly smaller than average lunar $\mathrm{npFe} \mathrm{e}^{0}$, while the $\mathrm{npFe} \mathrm{e}^{0}$ on Mercury appears to be significantly larger.

Acknowledgements: NASA support (NGT9-66, SKN), (NAG5-11763, CMP) and (RTOP 344-31-40-07, LPK) is gratefully acknowledged, as is support for SKN through the NASA Postdoctoral Program. Thank you to D. Mittlefehldt, NASA JSC, for all his help with getting the Fe-content data. We would also like to acknowledge C. Galindo, Hernandez Engineering, for doing the INAA, and B. Leinweber, Texas A\&M Nuclear Science Center, for doing the irradiation. Thank you to D. Dyar, Mt Holyoke, for providing the Mössbauer analysis and to M. 
Rutherford, Brown University, for his assistance in the lab and the use of his gas-flow oven. Thank you to S. Kunz, Brown U., and G. Robinson, NASA JSC, for their help with sample preparation and W. McCarthy at GRACEDavidson for providing some of the silica gels. All laboratory spectra were acquired using RELAB, a multi-user facility supported by NASA under NAG5-13609.

\section{Appendix:}

\section{Some notes on the properties of silica gel:}

While silica gels provide a reasonably spectrally-neutral background for us to observe the effects of $n p \mathrm{Fe}^{0}$, there are several interesting issues that have arisen with respect to the inherent properties of the silica gels themselves. These effects are worth noting, but are small and can be largely ignored for our purposes.

Particle size is known to strongly influence albedo and, as noted earlier, we were unable to attain silica gel starting material of the various pore sizes that were all the same particle sizes (see Table 1). These differences may make it difficult to compare albedo across suites. The shape of the continuum, though, is found to be largely unaffected by changes in particle size. To test the effect of particle size on individual silica gel grains, a sample of the SG6 gel was crushed to create a suite of size fractions. The sample was then impregnated with an intermediate amount of $\mathrm{npFe}^{0}(\sim 0.3 \mathrm{wt} \%)$. After iron reduction, the sample was dry sieved into six size fractions. In Fig. 13 is shown the reflectance spectra of the suite. As was expected from silicate samples (Adams and Filice, 1967), the smaller size fractions have higher albedo. The range of brightness observed, however, is smaller than that typical of this range of particle sizes for silicate samples. Much of the light scattering is thus occurring within the particles rather than at 
particle boundaries. After scaling to unity at $1300 \mathrm{~nm}$ (Fig. 13b), it can be seen that particle size of the silica gel host has only a minor effect on the spectral character of the continuum. There are nevertheless clear differences in the strength of the $\mathrm{OH}$ bands with the finer particles having smaller absorption bands. The spectra of the finer particles also appear to be slightly more curved throughout the visible region. The differences though, are small, particularly for the particle sizes utilized in our study (Table 1). These inherent particle size differences of the host material appear to be far overshadowed by the differences in $\mathrm{npFe}^{0}$ sizes.

For each of the four suites of silica gels, a null sample was included in the suite in which no iron was added, though the samples were exposed to the same heating and reducing conditions as the others. These no-iron-added samples provide an opportunity to examine the inherent spectral characteristics of the different gel suites. In Fig. 14 are shown spectra for these null samples scaled at $1300 \mathrm{~nm}$. The spectra do show slight variations. All four spectra exhibit a small but sharp downturn at wavelengths short of $350 \mathrm{~nm}$. There is also some variation between the samples in their general slope. The SG2.0 sample displays a slightly red slope while the other three samples have slightly blue slopes. The initial slope differences are not negligible, although they are quickly overwhelmed by the changes seen with the addition of $n p \mathrm{Fe}^{0}$.

\section{References:}

Adams, J.B. and Filice, A.L. 1967. Spectral Reflectance 0.4 to 2.0 Microns of Silicate Rock Powders. J. Geophys. Res., 72, 5705-5715.

Adams, J.B. and McCord, T.B. 1973. Vitrification darkening in the lunar highlands and identification of Descartes material at the Apollo 16 site. Proc. Lunar and Planet. Sci. Conf. $4^{\text {th }}, 163-177$. 
Allen, C.C., Morris, R.V. and McKay, D.S. 1996. An experimental analog to maturing lunar soil. (abstract) Lunar Planet. Sci. XXXVII, 13-14.

Basu, A. and McKay, D.S. 1983. Rarity of Lunar Soil Analogs in Meteorites. Meteoritics, 18, 263-264.

Blewett, D.T., Lucey, P.G., Hawke, B.R., Ling, G.G. and Robinson, M.S. 1997. A Comparison of Mercurian Reflectance and Spectral Quantities with those of the Moon. Icarus 129, 217231.

Binzel R.P., Bus S.J., Burbine T.H. and Sunshine J.M. 1996. Spectral Properties of Near-Earth Asteroids: Evidence for Sources of Ordinary Chondrite Meteorites. Science. 273, 946-948.

Binzel, R.P., Harris, A.W., Bus, S.J. and Burbine, T.H. 2001. Spectral Properties of Near-Earth Objects: Palomar and IRTF Results for 48 Objects Including Spacecraft Targets (9969) Braille and (10302) 1989 ML. Icarus, 151, 139-149.

Britt, D.T. and Pieters, C.M. 1994. Darkening in black and gas-rich ordinary chondrites: The spectral effects of opaque morphology and distribution. Geochem. Cosmochim. Acta, 58, 3905-3919.

Chapman, C.R. 2004. Space Weathering of Asteroid Surfaces. Ann. Rev. Earth and Planet. Sci., 32, 539-567.

Cintala, M.J. 1992. Impact-Induced Thermal Effects in the Lunar and Mercurian Regoliths. J. Geophys. Res., 97, 947-973.

Conel J.E. and Nash D.B. 1970. Spectral reflectance and albedo of Apollo 11 lunar samples: Effects of irradiation and vitrification and comparison with telescopic observations. Proceedings of the Apollo 11 Lunar Science Conference, 3, 2013-2023. 
Hapke, B. 1977. Interpretations of optical observations of Mercury and the Moon. Phys. Earth Planet. Interiors, 15, 264-274.

Hapke, B. 1986. On the Sputter Alteration of Regoliths of Outer Solar System Bodies. Icarus, 66, 270-279.

Hapke, B. 2001. Space Weathering from Mercury to the asteroid belt. J. Geophys. Res., 106, 10039-10073.

Hartmann, W.K. 1977. Relative Cater Production Rates on Planets. Icarus, 31, 260-276.

Haskin, L. and Warren, P. 1991. Lunar Chemistry. In: Heiken, G.H., Vaniman, D.T. and French, B.M. (eds.), Lunar Sourcebook, Cambridge Univ. Press, 357-474.

Housley R.M., Grant R.W. and Paton N.E. 1973. Origin and characteristics of excess Fe metal in lunar glass welded aggregates. Proc. of the $4^{\text {th }}$ Lunar Sci. Conf., 3, 2737-2749.

Horz, F., Cintala, M.J., See, T.H and Le, L. 2005. Shock melting of ordinary chondrite powders and implications for asteroidal regoliths. Meteoritics and Planet. Sci., 40, 1329-1346.

James, C.L., Basu, A., Wentworth, S.J. and McKay, D.S. 2001. Grain Size Distribution of Fe Globules in Lunar Agglutinitic Glass: First Results from Apollo 17 Soil 78421 (abstract). GSA Annual Mtg, Boston MA, 27433.

Jeanloz, R., Mitchell, D.L., Sprague, A.L. and de Pater, I. 1995. Evidence for a basalt-free surface on Mercury and implications for internal heat. Science, 268, 1455-1457.

Keller, L.P. and Clement, S.J. 2001. Formation of Nanophase iron in the lunar regolith. (abstract) Lunar Planet. Sci. XXXII, 2097.

Keller, L.P., Wentworth, S.J. and McKay, D.S. 1998. Space Weathering: Reflectance Spectroscopy and TEM Analysis of Individual Lunar Soil Grains. (abstract) Lunar Planet. Sci. XXXIX, 1762. 
Keller, L.P., Wentworth, S.J., Gezo, J., McKay, D.S., Taylor L.A., Pieters, C.M., and Morris, R.V. 1999. Space Weathering Alteration of Lunar Soil Grains. (abstract) Lunar Planet. Sci. XXX, 1820.

McCord, T.B. and Clark, R.N. 1979. The Mercury soil: Presence of $\mathrm{Fe}^{2+}$. J. Geophys. Res., 84, 7664-7668.

Mittlefehldt, D.W. 1994. The genesis of diogenites and HED parent body petrogenesis. Geochim. et Cosmochim. Acta 58, 1537-1552.

Morris, R.V. 1978. The surface exposure (maturity) of lunar soils: Some concepts and Is/FeO compilation, Proc. Lunar Planet. Sci. Conf. 9th, 2278-2297.

Morris, R.V., Agresti, D.G., Lauer, H.V. Jr., Newcomb, J.A., Shelfer, T.D. and Murali, A.V. 1989. Evidence for Pigmentary Hematite on Mars Based on Optical, Magnetic, and Mossbauer Studies of Superparamagnetic (Nanocrystalline) Hematite. J. Geophys. Res., 94, B3, 2760-2778.

Noble, S.K. and Pieters, C.M. 2003. Space Weathering on Mercury: Implications for Remote Sensing. Astron Vestnik, 37, 34-39. (English version in Solar System Research 37(1), 31-35.)

Noble, S.K., Pieters, C.M., Taylor, L.A., Morris, R.V., Allen, C.C., McKay, D.S. and Keller, L.P. 2001. The Optical Properties of the Finest Fraction of Lunar Soil: Implications for Space Weathering. Meteoritics and Planet. Sci., 36, 31-42.

Noble, S.K., Pieters, C.M. and Keller, L.P. 2004. Quantitative aspects of space weathering: Implications for regolith breccia meteorites and asteroids. (abstract) Lunar Planet. Sci. XXXV, 1301. 
Noble, S.K., Pieters C.M., Hiroi T., and Taylor L.A. 2006. Using the modified Gaussian model to extract quantitative data from lunar soils, J. Geophys. Res., 111, E11009, doi:10.1029/2006JE002721.

Pieters, C.M., Fischer, E.M., Rode, O. and Basu, A. 1993. Optical Effects of Space Weathering: The Role of the Finest Fraction. J. Geophys. Res., 98, 20,817-20,824.

Pieters, C.M., Rode, O., Fischer, E.M. and Basu, A. 1994. Lunar space weathering and the optical properties of lunar regolith. Astron Vestnik, 28, 109-119.

Pieters, C.M., Taylor, L.A., Noble S.K., Keller, L.P., Hapke B., Morris, R.V., Allen, C.C., McKay D.S. and Wentworth S. 2000. Space weathering on airless bodies: Resolving a mystery with lunar samples. Meteoritics and Planetary Science, 35, 1101-1107.

Sasaki, S. and Kurahashi E. 2004. Space Weathering on Mercury. Adv. in Space Res., 33, 21522155.

Sprague, A.L., Warell, J., Emery, J., Long, A. and Kozlowski, R.W.H. 2004. Mercury: First spectra from 0.7 to $5.5 \mu \mathrm{m}$ support low $\mathrm{FeO}$ and feldspathic composition. (abstract) Lunar Planet. Sci. XXXV, 1630.

Taylor, L.A., Pieters, C.M., Morris, R.V., Keller, L.P. and McKay D.S. 2001. Lunar mare soils: Space weathering and the major effects of surface-correlated nanophase Fe. J. Geophys. Res. 106, $27,985-28,000$.

Vilas, F. 1985. Mercury: Absence of crystalline $\mathrm{Fe}^{2+}$ in the regolith. Icarus, 64, 133-138.

Vilas, F., Leake, M.A. and Mendell, W.W. 1984. The Dependence of Reflectance Spectra of Mercury on Surface Terrain. Icarus, 59, 60-68.

Warell, J. 2002. Properties of the Hermean regolith: III. Disk-resloved vis-NIR reflectance spectra and implications for the abundance of iron. Icarus, 161, 199-222. 
Zangwill, A. 1988. Physics at Surfaces. Cambridge Univ. Press, Cambridge MA. 
Table 1. Silica gels used in this study and resulting dispersed $n p F e^{0}$

\begin{tabular}{|l|c|c|c|c|}
\hline \hline Name & Particle size & Pore size & \multicolumn{2}{|c|}{$\mathrm{npFe}^{0}$ size } \\
\cline { 4 - 5 } & & & nominal range & average \\
\hline SG2 $^{*}$ & $125-250 \mu \mathrm{m}$ & $2.3 \mathrm{~nm}$ & $5-15 \mathrm{~nm}$ & 8 \\
\hline SG6 $^{*}$ & $75-150 \mu \mathrm{m}$ & $6 \mathrm{~nm}$ & $10-25 \mathrm{~nm}$ & 15 \\
\hline SG25 $^{*}$ & $75-150 \mu \mathrm{m}$ & $25 \mathrm{~nm}$ & $25-50 \mathrm{~nm}$ & 35 \\
\hline SG50 $^{\#}$ & $30-75 \mu \mathrm{m}$ & $50 \mathrm{~nm}$ & $20-200 \mathrm{~nm}$ & 40 \\
\hline
\end{tabular}

*Manufactured by GRACEDavidson

"Manufactured by Mallinckrodt Baker Inc. 
Table 2. Iron concentration measured for prepared silica gels

\begin{tabular}{|l|c|c|}
\hline & \multicolumn{2}{|c|}{ Fe } \\
\hline Sample \# & wt $\%$ & \pm \\
\hline SG2.0 & 0.00508 & 0.00010 \\
\hline SG2.1 & 0.01822 & 0.00020 \\
\hline SG2.22 & 0.1091 & 0.0010 \\
\hline SG2.37 & 0.1651 & 0.0015 \\
\hline SG2.42 & 0.373 & 0.004 \\
\hline SG2.43 & 0.539 & 0.005 \\
\hline SG2.5 & 1.209 & 0.010 \\
\hline SG2.6 & 1.646 & 0.014 \\
\hline SG6.0 & 0.00488 & 0.00009 \\
\hline SG6.1 & 0.01868 & 0.00022 \\
\hline SG6.2 & 0.0690 & 0.0007 \\
\hline SG6.22 & 0.1329 & 0.0016 \\
\hline SG6.22 & 0.1334 & 0.0013 \\
\hline SG6.23 & 0.1921 & 0.0021 \\
\hline SG6.3 & 0.297 & 0.003 \\
\hline SG6.5 & 2.112 & 0.019 \\
\hline SG25.0 & 0.00550 & 0.00013 \\
\hline SG25.1 & 0.02382 & 0.00026 \\
\hline SG25.2 & 0.2024 & 0.0018 \\
\hline SG25.23 & 0.3359 & 0.0028 \\
\hline SG25.23 & 0.348 & 0.003 \\
\hline SG25.3 & 0.580 & 0.005 \\
\hline SG25.4 & 0.940 & 0.009 \\
\hline SG25.5 & 3.780 & 0.020 \\
\hline SG50.0 & 0.00799 & 0.00017 \\
\hline SG50.1 & 0.02516 & 0.00028 \\
\hline SG50.12 & 0.0794 & 0.0009 \\
\hline SG50.15 & 0.0962 & 0.0010 \\
\hline SG50.22 & 0.1505 & 0.0018 \\
\hline SG50.23 & 0.1845 & 0.0020 \\
\hline SG50.4 & 0.498 & 0.005 \\
\hline SG50.5 & 1.895 & 0.016 \\
\hline SG50.5 & 1.885 & 0.016 \\
\hline
\end{tabular}




\section{Figure Captions:}

Figure 1. BSE image of sample from the SG6 suite showing the grain-to-grain and intra-grain variability in iron content. The greater the $\mathrm{npFe}^{0}$ content, the brighter the grain appears in backscatter.

Figure 2. Example TEM bright field images of each of the four powders used in this study: a) SG2 b) SG6 c) SG25 d) SG50. The porous texture of the powders is readily apparent. The $\mathrm{npFe}^{0}$ is black.

Figure 3. Examples of several anomalously large $n \mathrm{nFe}^{0}$-particles: Such particles, while uncommon, were identified in all four silica gel types. a) A small cluster of large particles on edge of grain from SG2 suite. b) Several large irregular particles along an apparent defect in the silica gel of a grain from SG6 suite. c) Two very large particles on the edge of a grain from the SG50 suite. d) A single large particle in the interior of a grain from the SG50 suite.

Figure 4. Mössbauer spectra of the highest-npFe ${ }^{0}$ sample from SG25 suite compared to an alpha-Fe foil and a hematite sample (Mössbauer data courtesy M. Darby Dyar).

Figure 5. Reflectance spectra and scaled reflectance spectra of the four silica gel suites: a) SG2 suite, b) SG6 suite, c) SG25 suite, d) SG50 suite.

Figure 6. Reflectance spectra of samples from each of the four suites with similar iron contents: a) $0.02 \mathrm{wt} \% \mathrm{Fe}^{0}$, and b) $0.2 \mathrm{wt} \% \mathrm{Fe}^{0}$.

Figure 7. Reflectance spectra of the results of mixing in the SG6 suite. The no-iron-added sample (SG6.0) was mixed in varying proportions with the most iron-rich sample (SG6.5 - $2.1 \mathrm{wt}$ $\% \mathrm{Fe})$. The entire SG6 suite is shown in gray for comparison.

Figure 8. Reflectance spectrum of the results of mixing roughly equal proportions of samples from the SG6 and SG25 suites, each containing similar amount of npFe ${ }^{0}$, roughly $0.2 \mathrm{wt} \%$.

Figure 9. Reflectance spectra of silica gels from the SG2, SG6 and SG25 suites versus the $<45$ $\mu \mathrm{m}$ fraction of three lunar soils, scaled to unity at $550 \mathrm{~nm}$. Lunar soil 14141 is a very immature soil with an $\mathrm{I}_{s} / \mathrm{FeO}$ value of 6 , soil 64801 is a mature highland soil $\left(\mathrm{I}_{\mathrm{s}} / \mathrm{FeO}=82\right)$, and soil 15071 is a submature mare soil $\left(\mathrm{I}_{\mathrm{s}} / \mathrm{FeO}=52\right)$. The iron contents $\left(\mathrm{wt} \% \mathrm{npFe}^{0}\right)$ of the silica gel samples are labeled.

Figure 10. TEM bright field image of a typical $\mathrm{npFe}^{0}$-bearing rim surrounding a grain of lunar soil 10084 (a), and compared to a typical region of agglutinitic glass (b). The $n p F e^{0}$ in agglutinates is, on average, considerably larger than the sizes seen in rims.

Figure 11. Reflectance spectra of silica gels from the SG2, SG6 and SG25 suites versus telescopic spectra from Mercury scaled to unity at $550 \mathrm{~nm}$. The Mercury spectra (Vilas et al., 1984) represent areas dominated by intercrater plains (Sept 74) and smooth plains plus the Caloris basin (Oct 74). The iron contents ( $\left.\mathrm{wt} \% \mathrm{npFe}^{0}\right)$ of the silica gel samples are labeled. 
Figure 12. Reflectance spectra of silica gels from the SG2, SG6 and SG25 suites versus two near earth asteroids (NEA) scaled to unity at $550 \mathrm{~nm}$. NEA spectra from Binzel et al. (2001) were obtained online, courtesy MIT (http://smass.mit.edu/). The iron contents $\left(\mathrm{wt} \% \mathrm{npFe} \mathrm{p}^{0}\right.$ ) of the silica gel samples are labeled.

Figure 13. Reflectance spectra (a) and scaled reflectance spectra (b) of an SG6 powder with $\sim 0.3 \mathrm{wt} \% \mathrm{npFe}{ }^{0}$ sieved into several size fractions to demonstrate the effects of changing particle size.

Figure 14. Reflectance spectra of powders processed with no iron added. These silica gels shown small but nontrivial differences in their reflectance spectra before the addition of $\mathrm{nFe}^{0}$. 


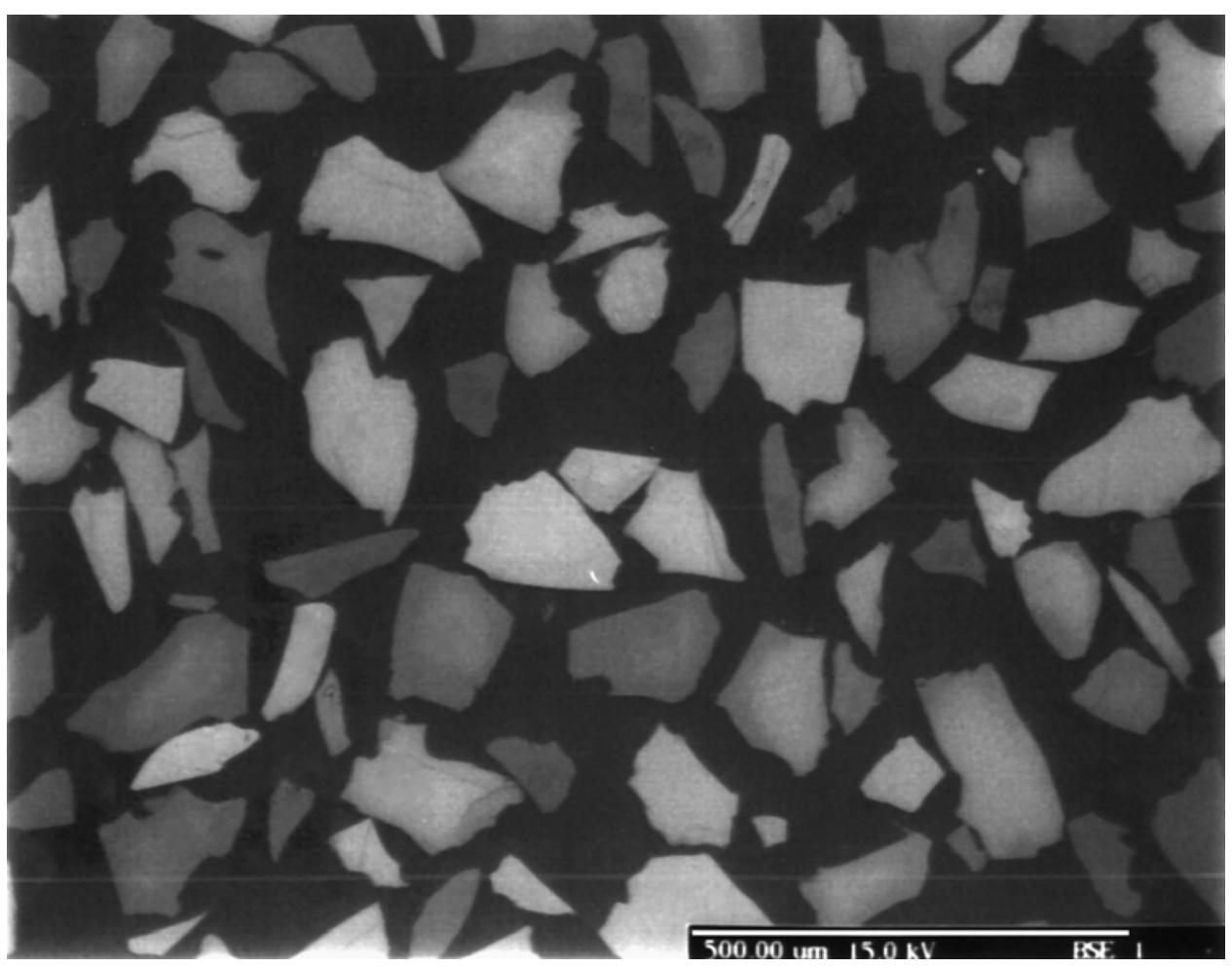

Figure 1.

$-35-$ 

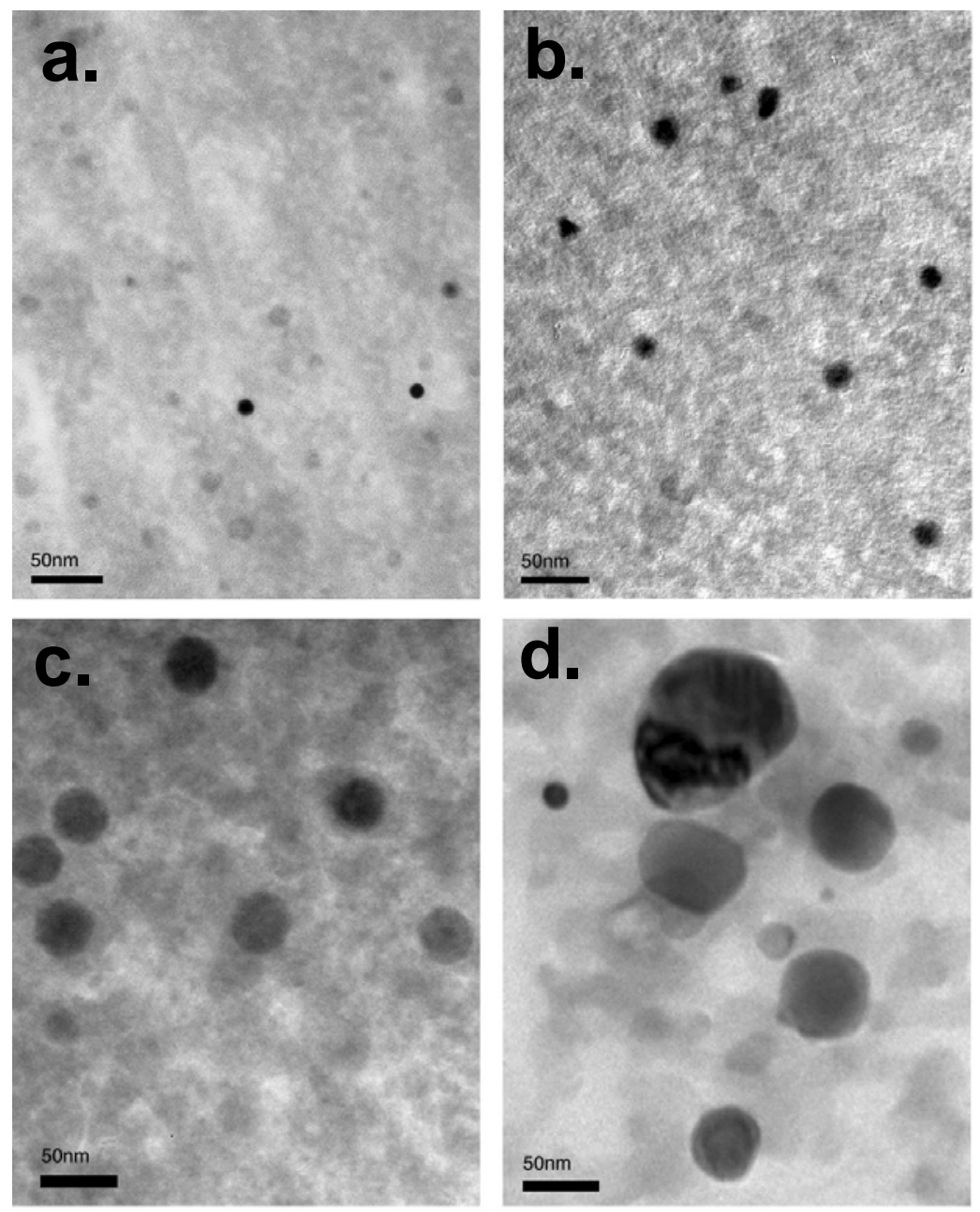

Figure 2.

- 36 - 


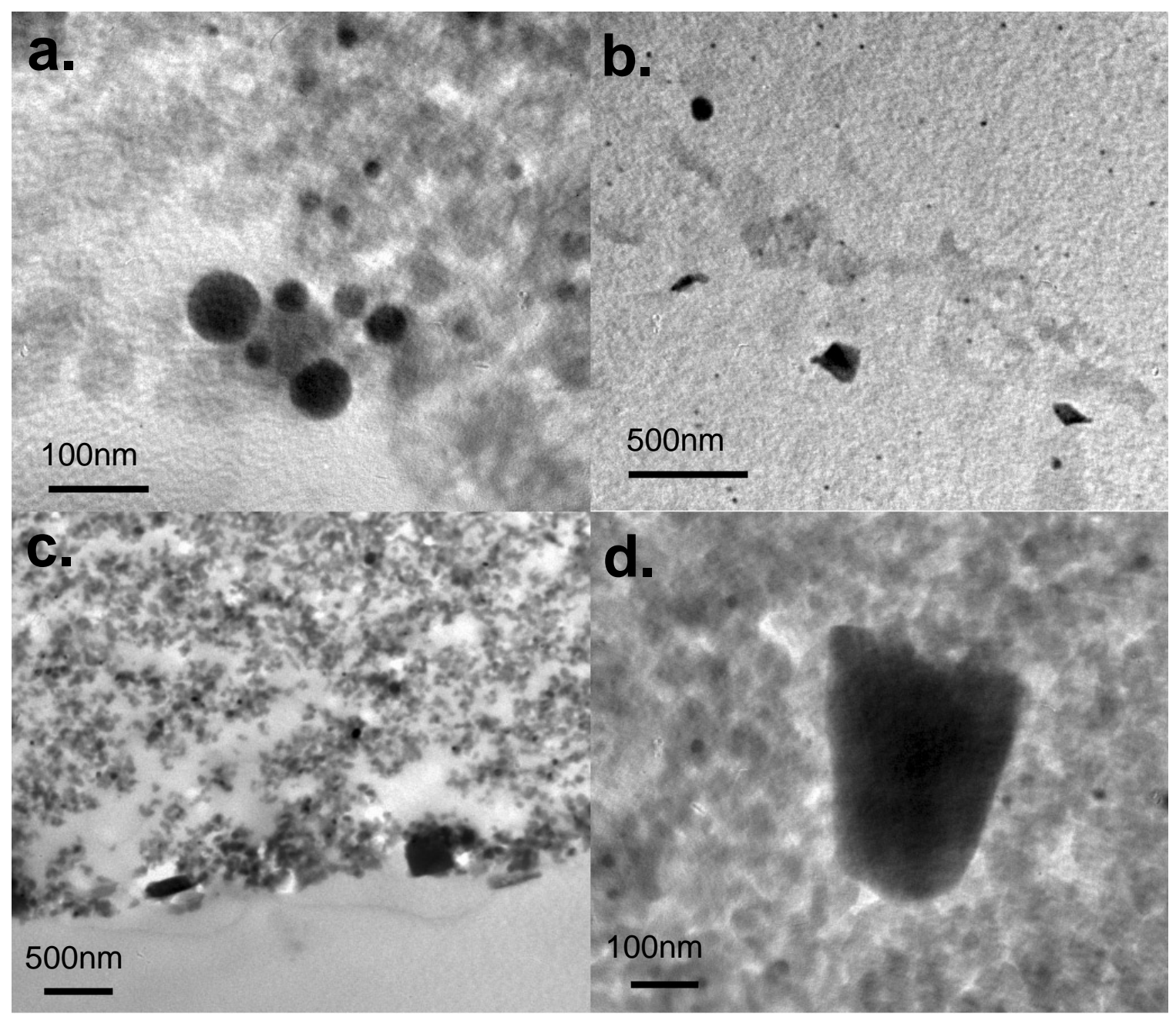

Figure 3. 


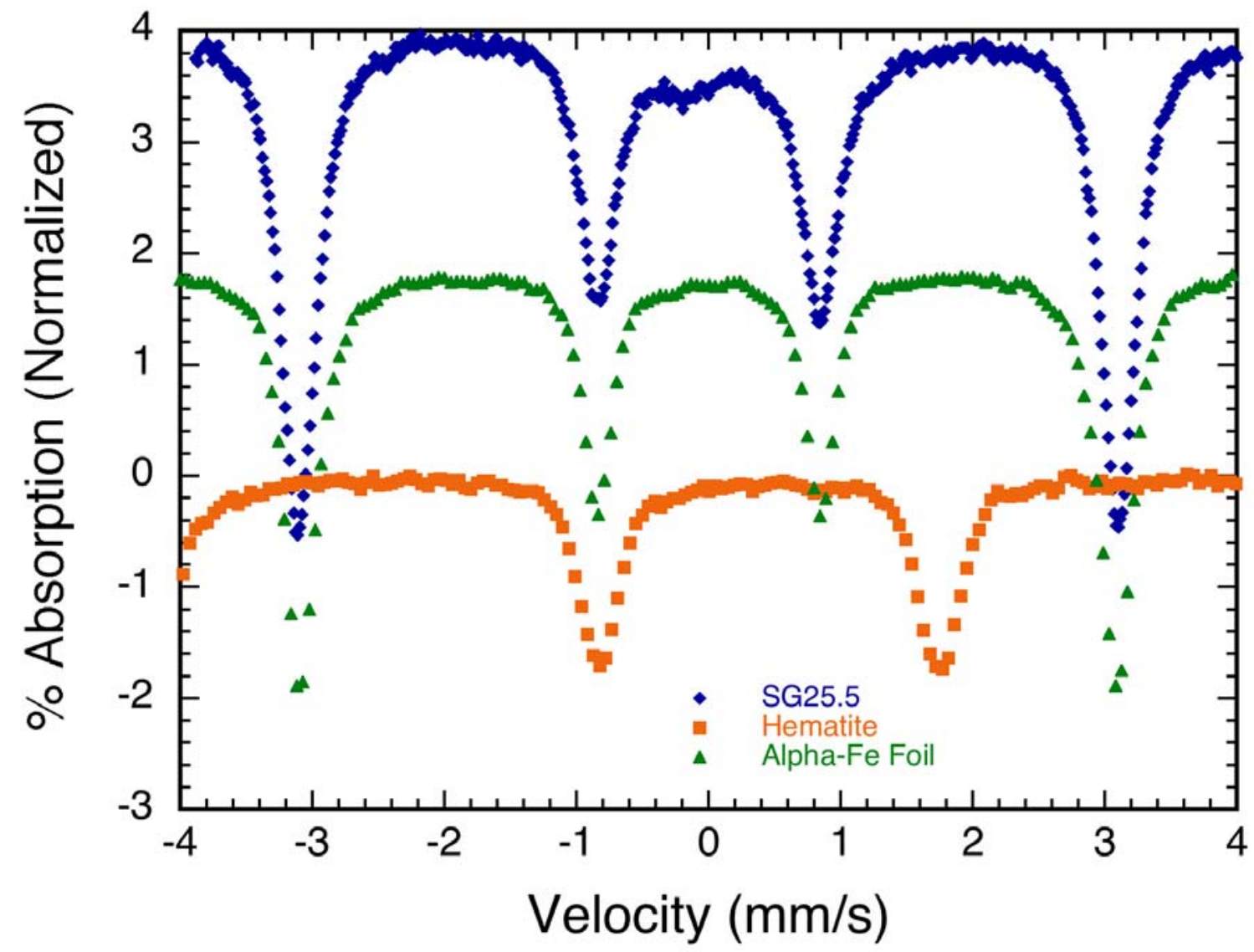

Figure 4. 

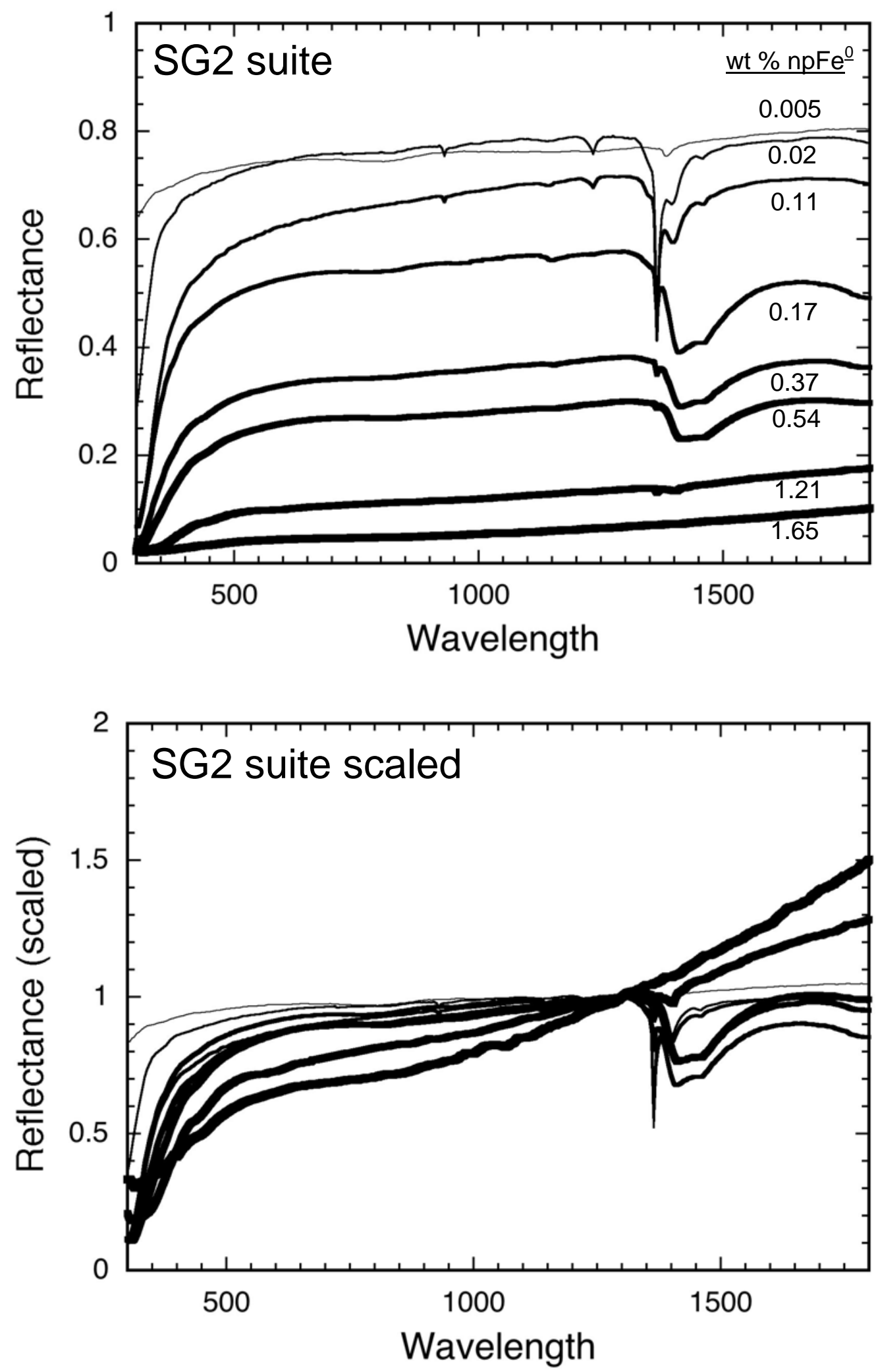

Figure 5a. 

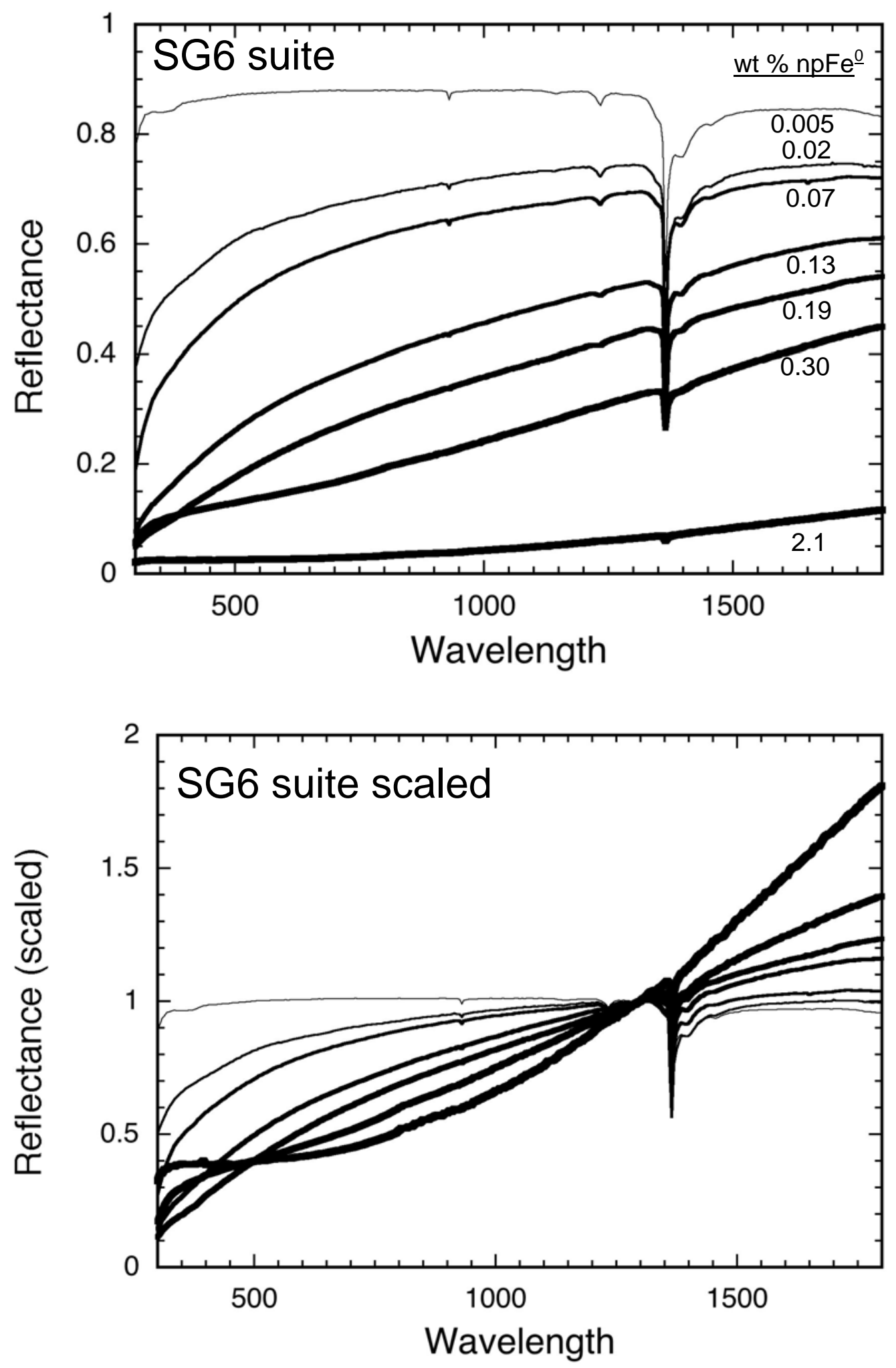

Figure 5b. 

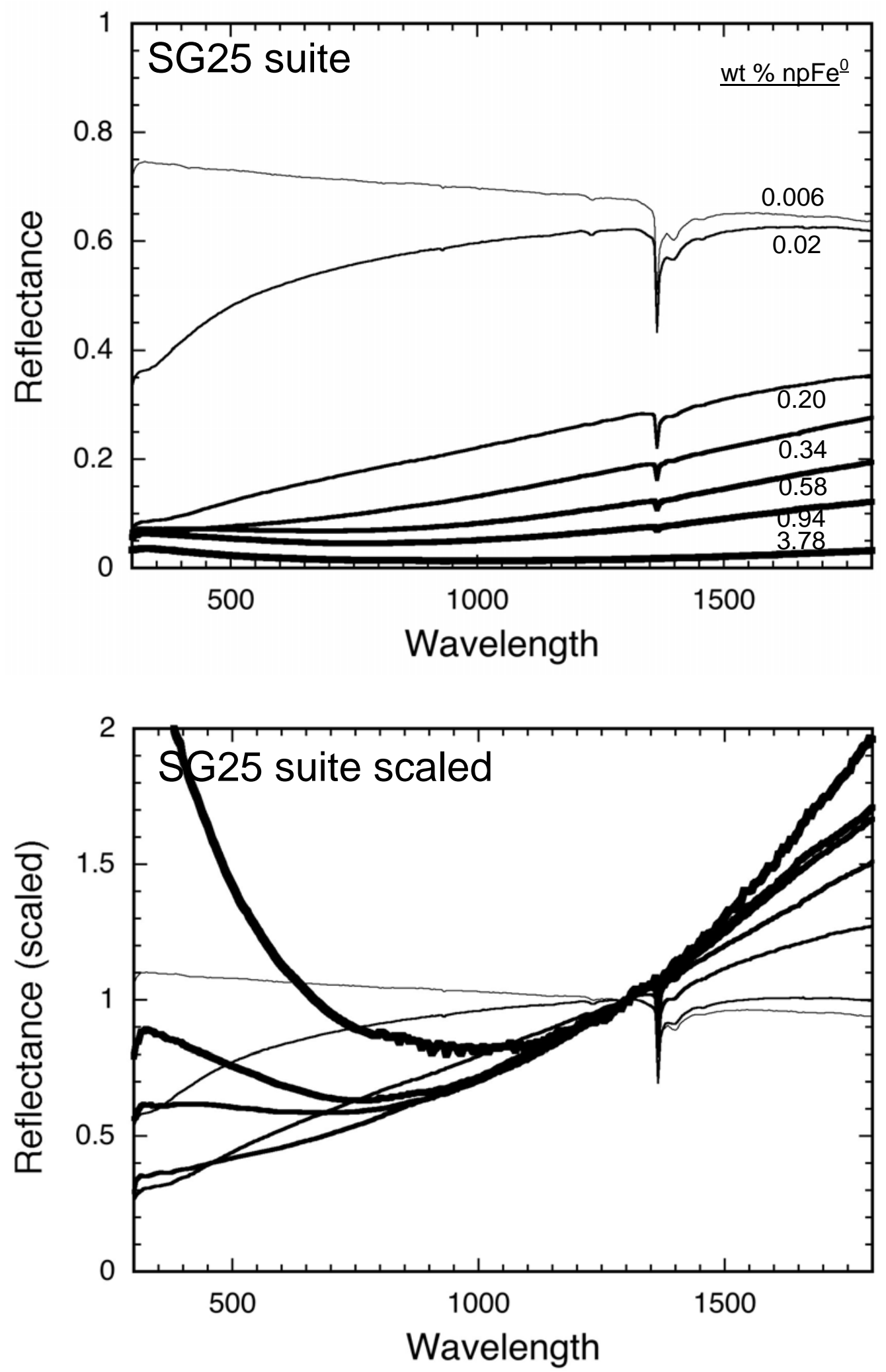

Figure 5c. 

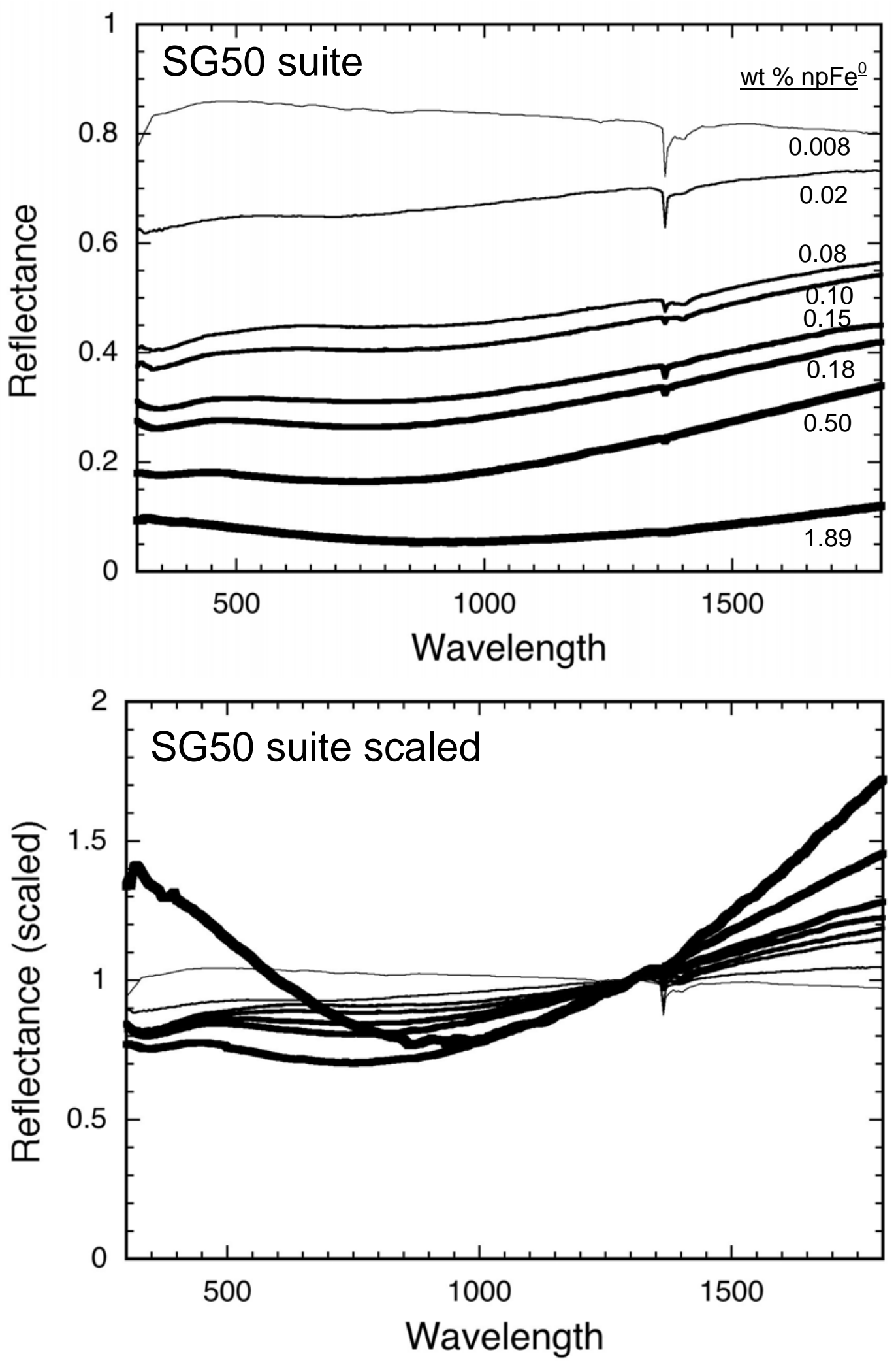

Figure 5d. 

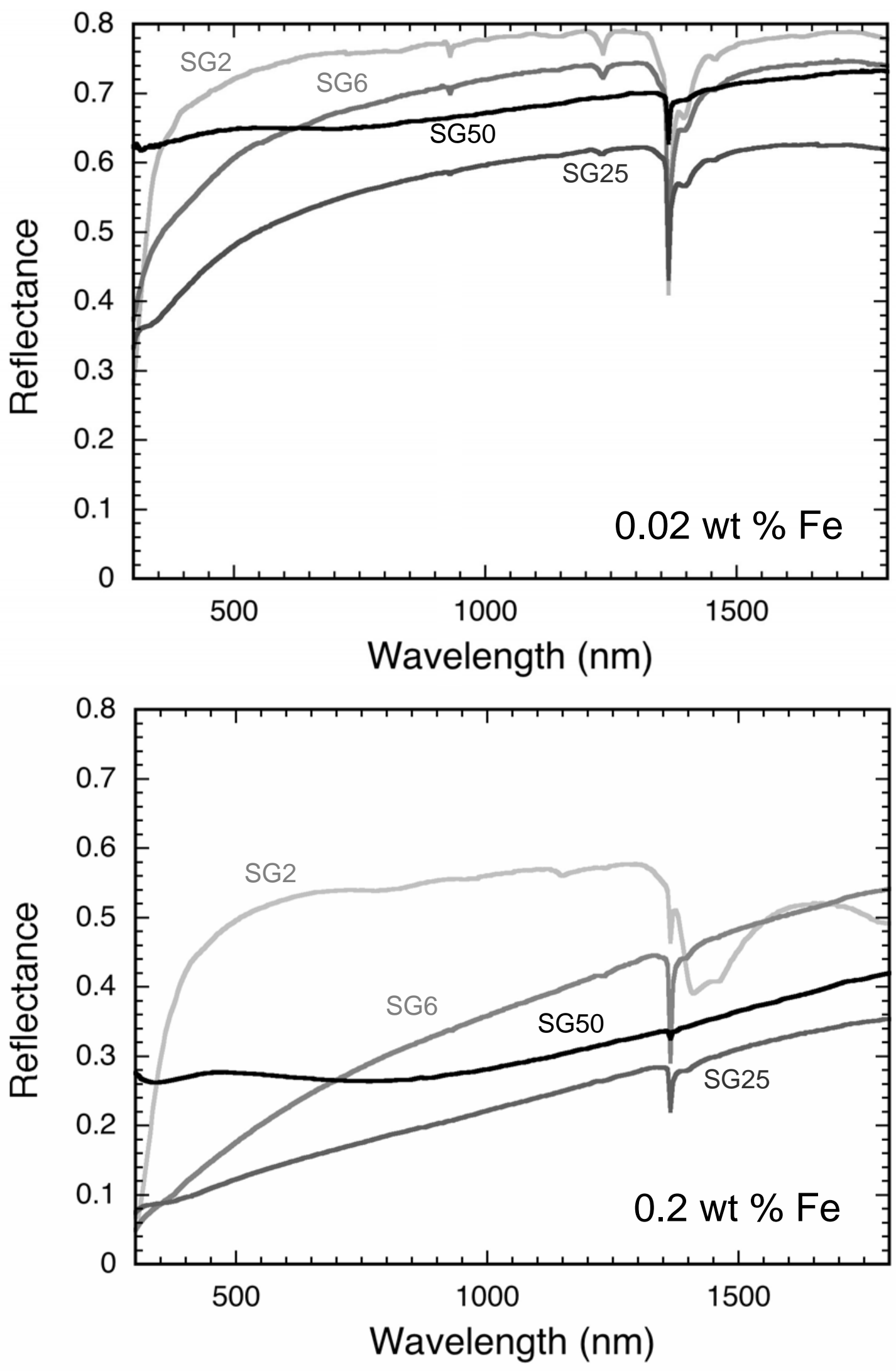

Figure 6. 


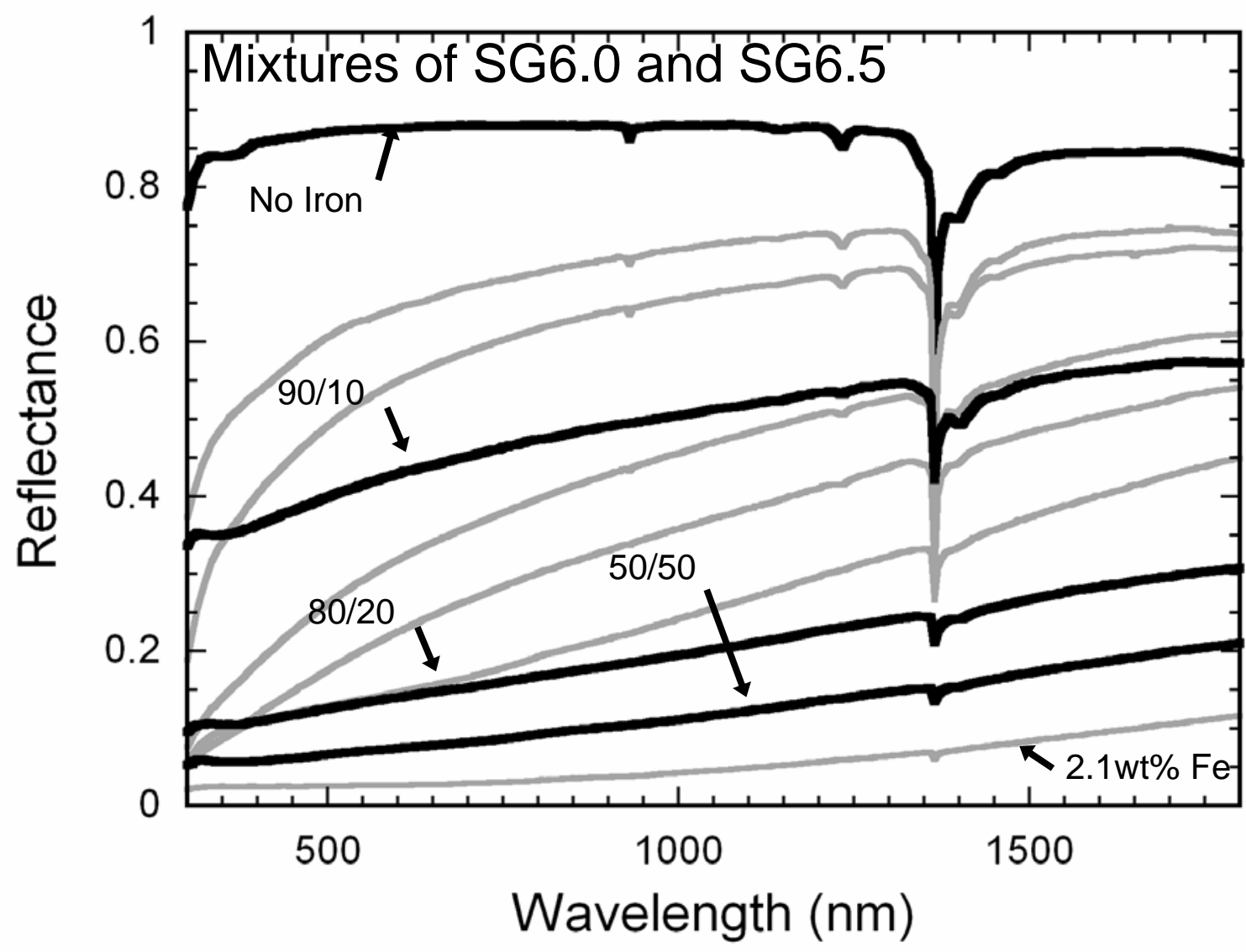

Figure 7. 


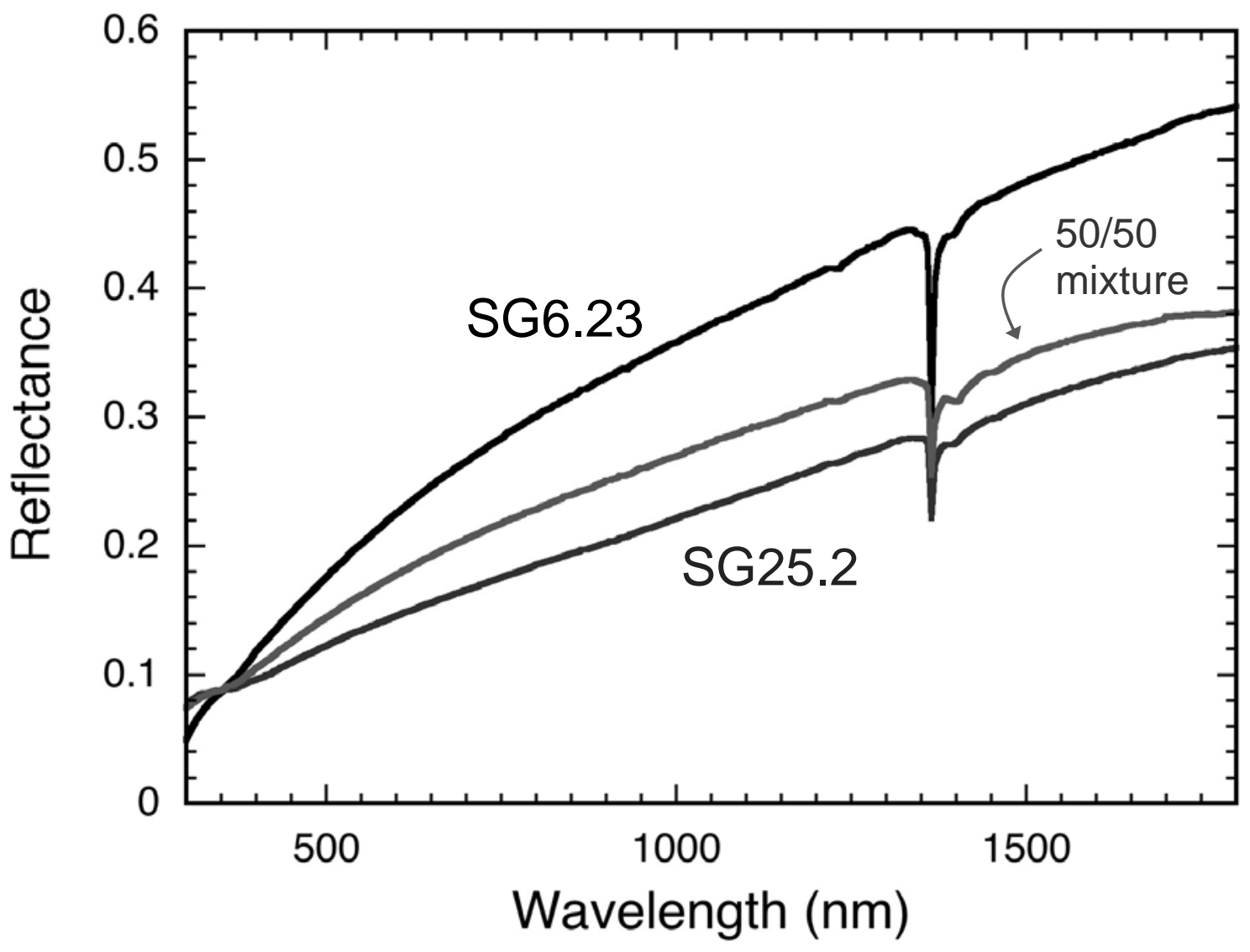

Figure 8. 


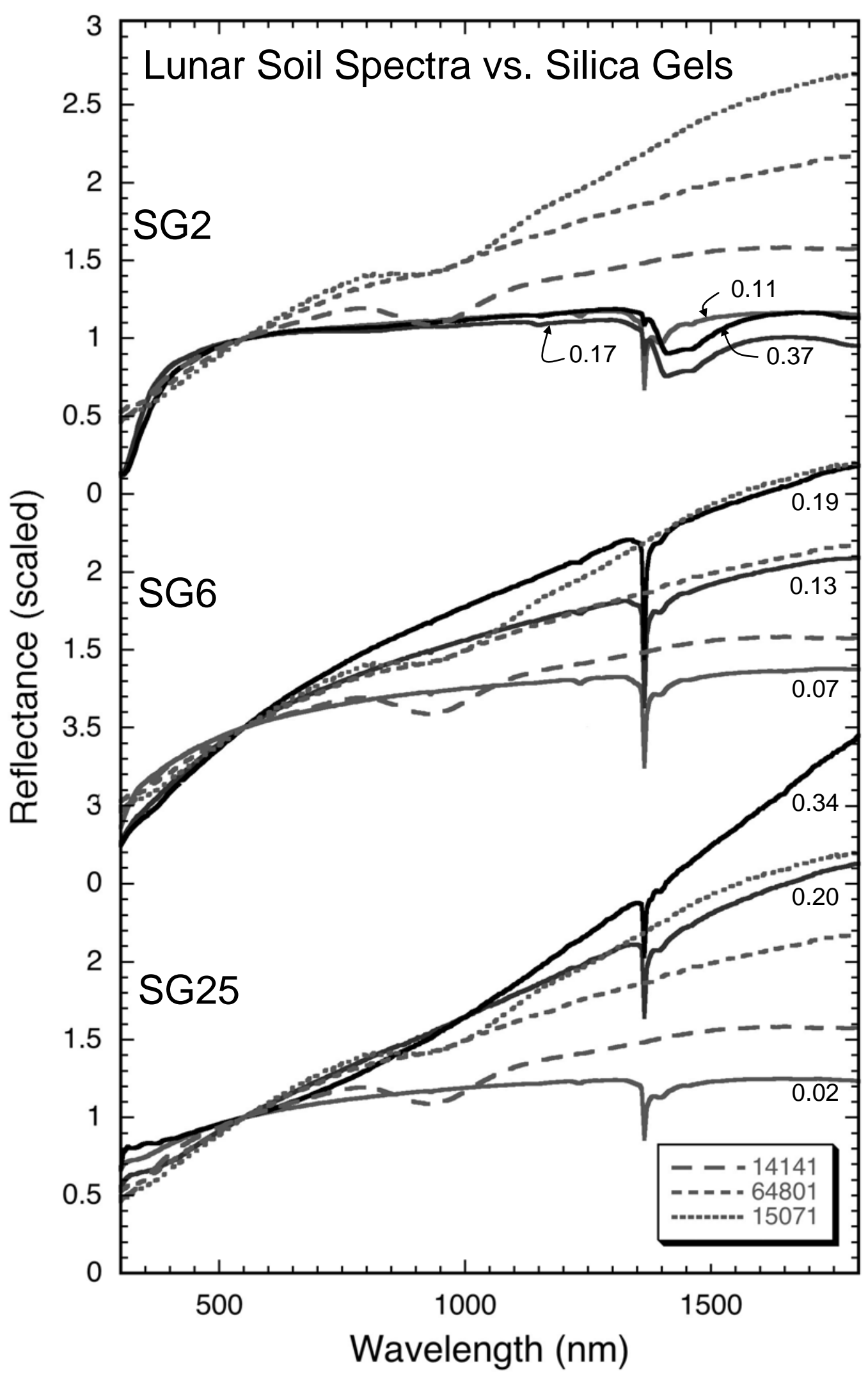

Figure 9. 


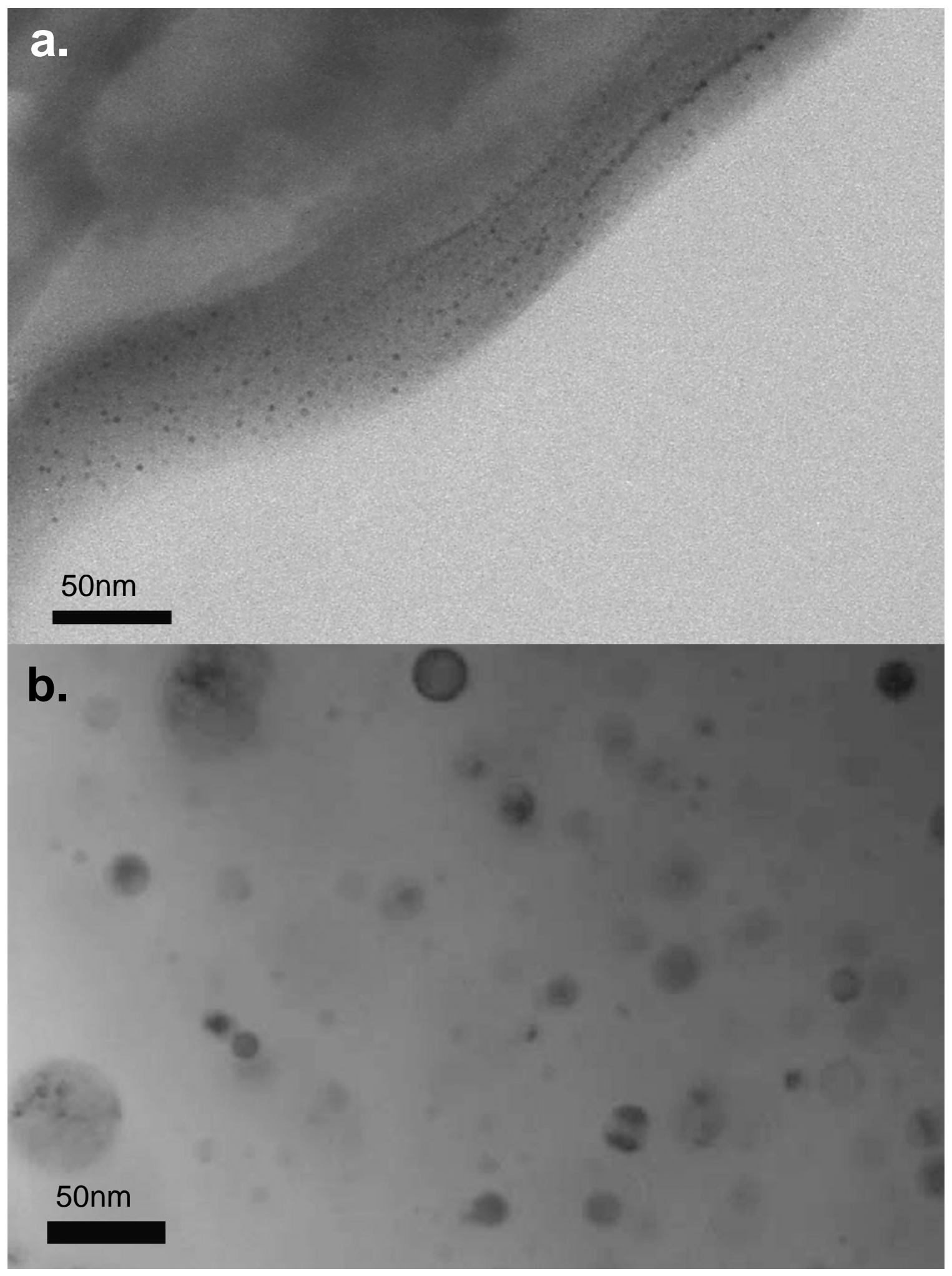

Figure 10.

- 47 - 


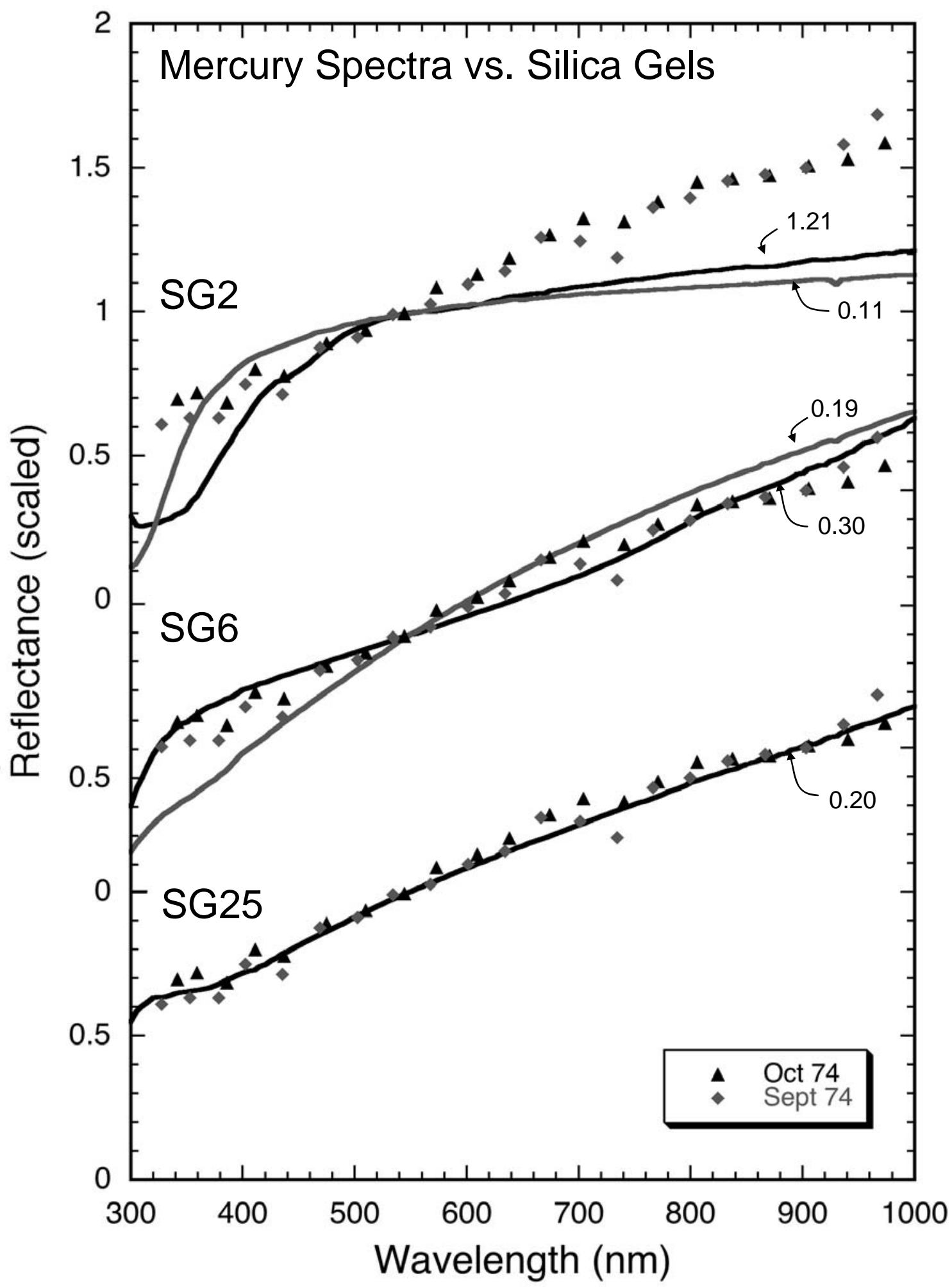

Figure 11. 


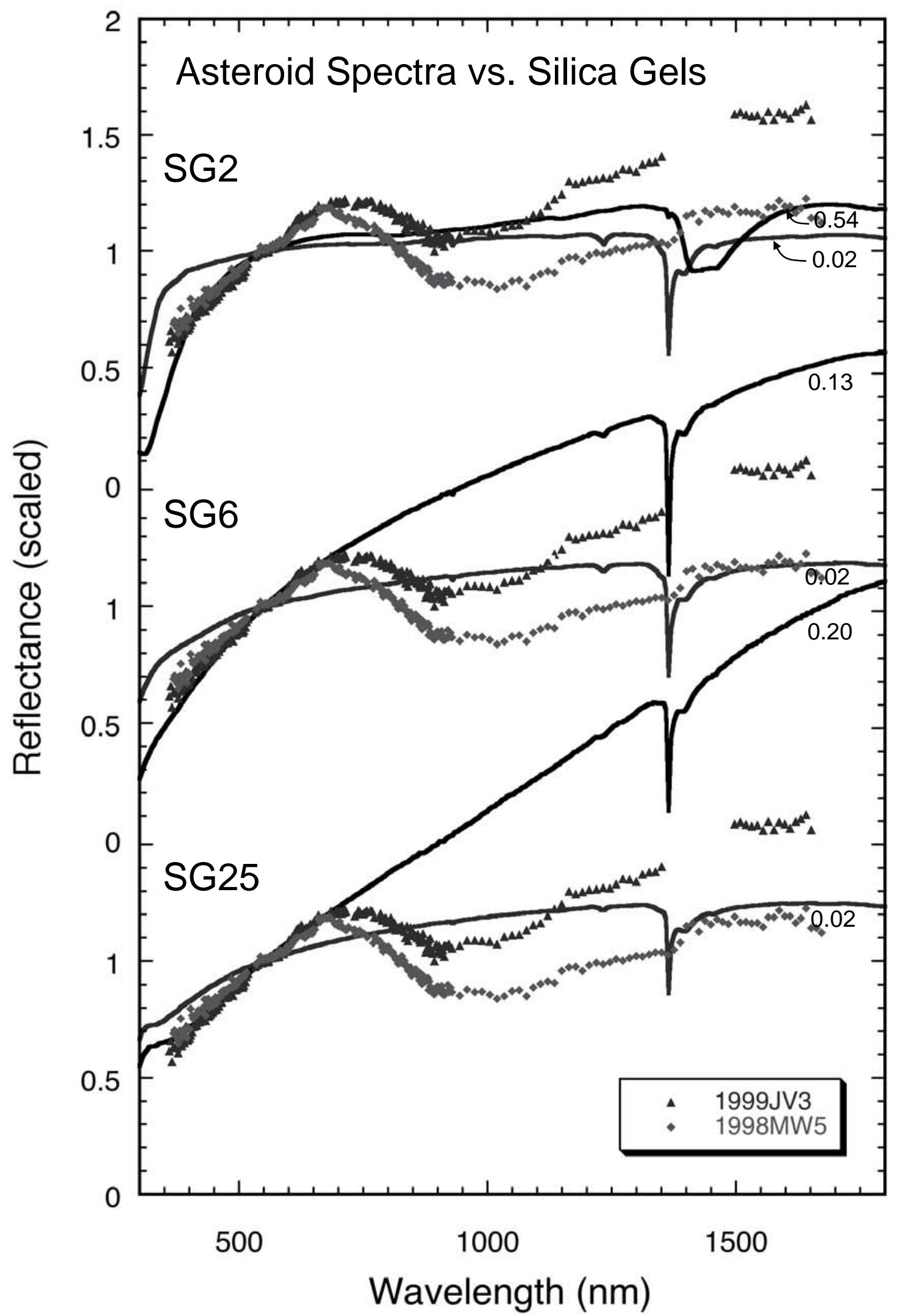

Figure 12. 

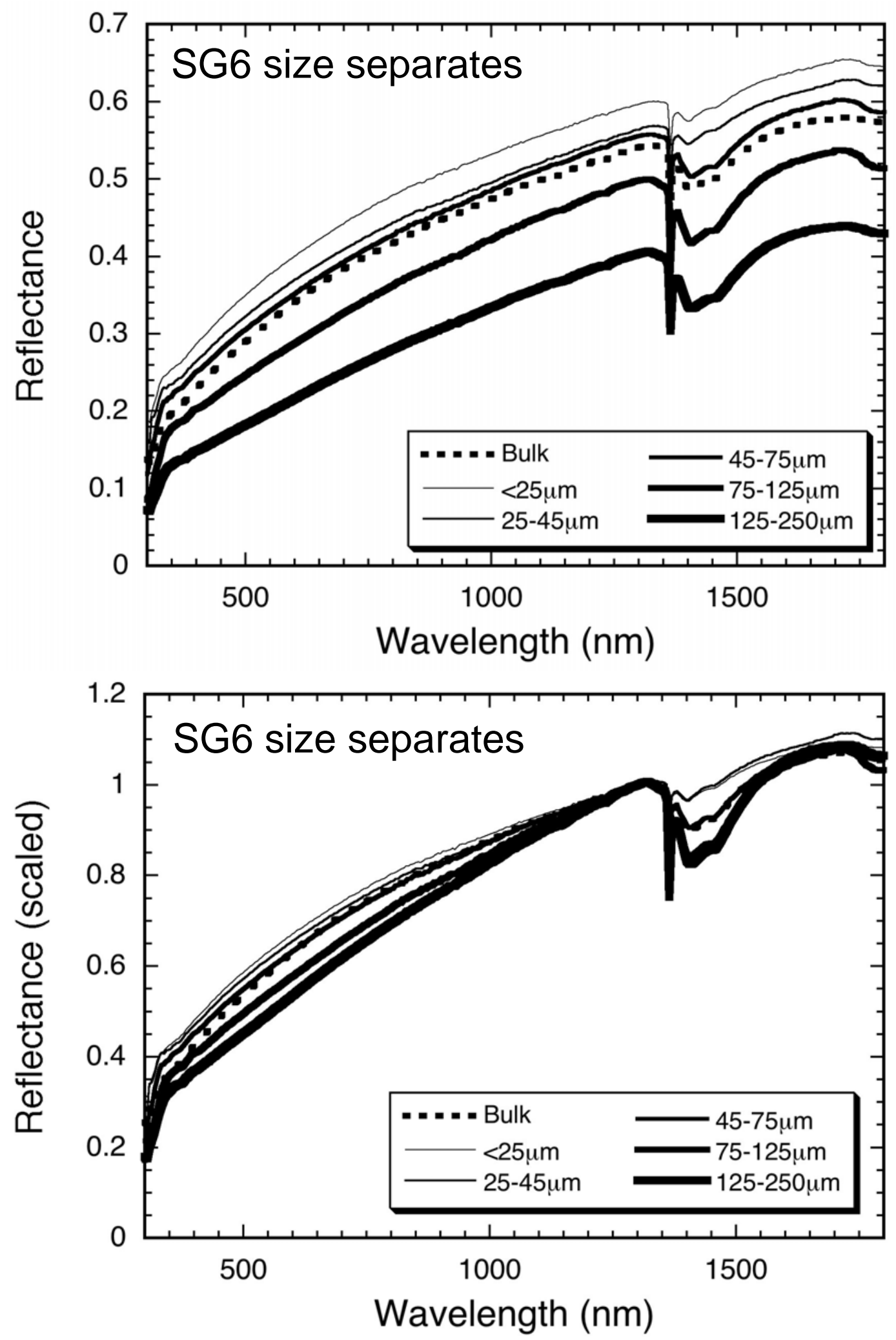

Figure 13. 


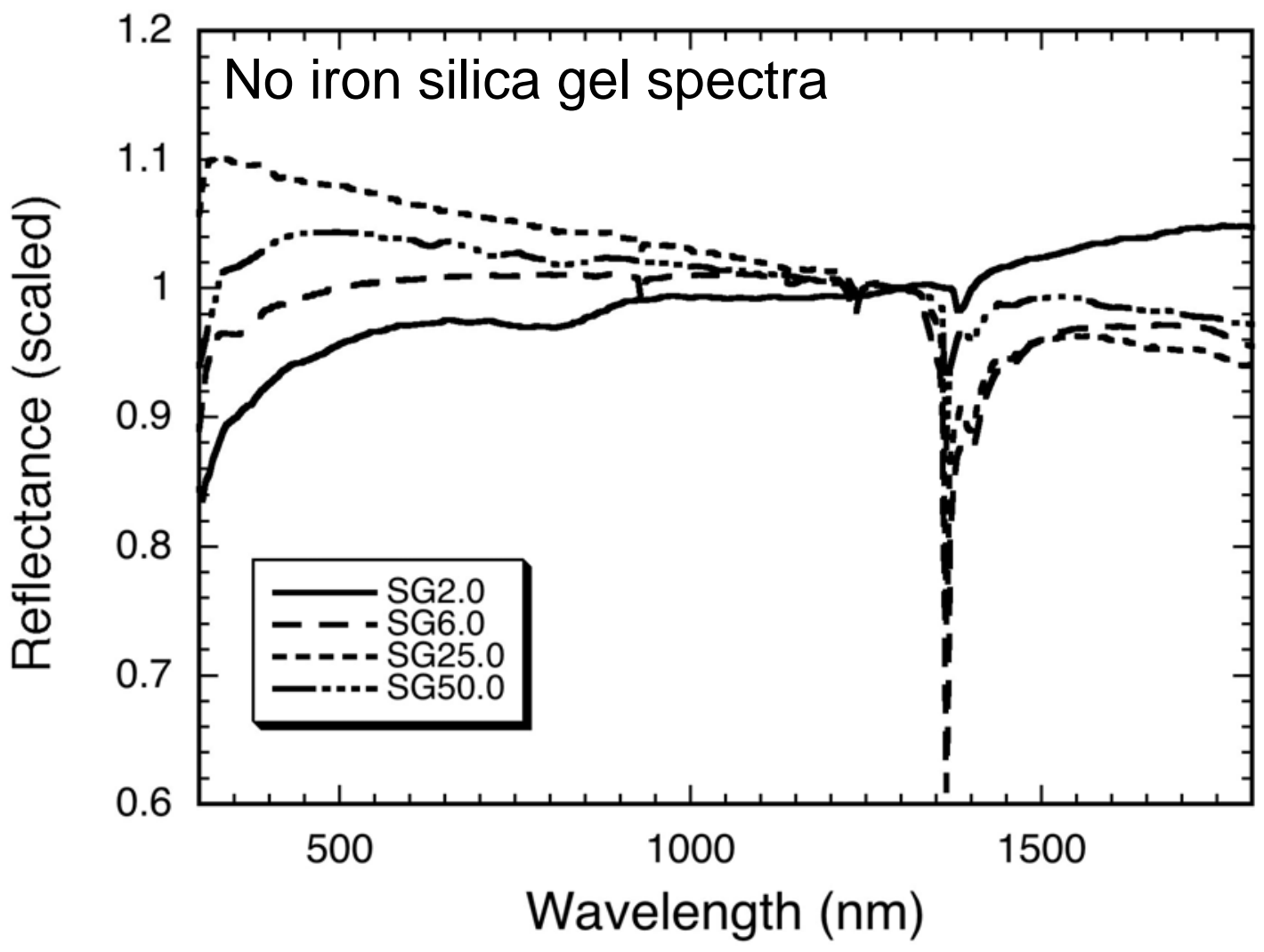

Figure 14. 